TRANSACTIONS OF THE

AMERICAN MATHEMATICAL SOCIETY

Volume 362, Number 6, June 2010, Pages 2923-2962

S 0002-9947(10)04969-X

Article electronically published on January 21, 2010

\title{
BANDO-FUTAKI INVARIANTS ON HYPERSURFACES
}

\author{
CHIUNG-JU LIU
}

\begin{abstract}
In this paper, the Bando-Futaki invariants on hypersurfaces are derived in terms of the degree of the defining polynomials, the dimension of the underlying projective space, and the given holomorphic vector field. In addition, the holomorphic invariant introduced by Tian and Chen (2002) is proven to be the Futaki invariant on compact Kähler manifolds with positive first Chern class.
\end{abstract}

\section{Contents}

1. Introduction

2. Bando-Futaki invariants

2.1. Curvature tensor on hypersurfaces

2.2. Chern forms

2.3. Computation of the Bando-Futaki invariants 2939

3. Chen and Tian's holomorphic invariants 2955

4. Appendix

Acknowledgements

References

\section{INTRODUCTION}

In 1983, Futaki introduced the well-known Futaki invariant [5], which is an obstacle to the existence of Kähler-Einstein metrics on a compact complex manifold with positive first Chern class. Other generalizations of the Futaki invariant were introduced later, all of which are obstructions to certain geometric structures. The Calabi-Futaki invariant [2] is an obstruction to the existence of Kähler metrics of constant scalar curvature on a compact Kähler manifold. The Bando-Futaki invariants, raised by Bando 1 in 1983, are obstructions to the harmonicity of the higher order Chern forms. The Bando-Futaki invariants vanish if and only if the short-time solutions of the almost Kähler-Einstein metrics exist (cf. Leung [11). Tian and Zhu found a holomorphic invariant [16], which is an obstruction to the existence of a Kähler-Ricci soliton. Recently, Futaki [7] generalized the BandoFutaki invariants and the Futaki-Morita invariants [8]. The new invariants give

Received by the editors December 21, 2007.

2010 Mathematics Subject Classification. Primary 32Q15; Secondary 53C55.

Key words and phrases. Bando-Futaki invariants, Futaki invariants.

The author was partially supported by NSF:DMS-0202508 and NSF:DMS-0347033 during her Ph.D. study.

(c) 2010 American Mathematical Society Reverts to public domain 28 years from publication 
obstructions to asymptotic Chow semi-stability when the invariant polynomials are Todd polynomials.

Efficient methods for computing the Futaki invariant and the generalized Futaki invariants are essential to characterizing the existence of certain geometric structures. $\mathrm{Lu}[12$ constructed a formula to evaluate the Futaki invariant on complete intersections. The formula depends on the dimension of the projective space, the degree of the defining polynomials, and the given tangent holomorphic vector field. Concurrently, Yotov [17 derived the same result with a different approach. On complete intersections, Phong and Sturm 14] formularized the Futaki invariant and the Mabuchi energy functional using the Deligne pairing. Their methods may lead to a complete solution to the problem of computing the Futaki invariant.

The main part of this paper is the computation of the Bando-Futaki invariants on hypersurfaces in $\mathbb{C P}^{n}$. The value, stated in Theorem 1.1, is in terms of the dimension $n$, the degree of the defining polynomial of the hypersurface, and the tangent vector field. In Theorem 1.2, we prove that Chen and Tian's holomorphic invariants introduced in [3, section 5] are the Futaki invariants.

Let $M$ be an $m$-dimensional compact complex manifold with positive first Chern class $c_{1}(M)>0$. Let $\omega_{M}$ be a Kähler form on $M$ such that $\left[\omega_{M}\right] \in c_{1}(M)$. Let $\Theta$ be the curvature tensor with respect to the Kähler metric $\omega_{M}$ on the holomorphic tangent bundle $T^{1,0}(M)$. Let $c_{q}(\Theta)=P^{q}(\Theta)$ denote the $q$-th Chern form of $\Theta$, where $P^{q}(\Theta)$ are the elementary polynomials defined by

$$
\operatorname{det}(\Theta+t \cdot I)=\sum_{q=0}^{m} P^{q}(\Theta) \cdot t^{m-q} .
$$

Define the Chern classes $c_{q}(M)$ by

$$
c_{q}(M)=\left[P^{q}(\Theta)\right] \in H_{D R}^{2 q}(M),
$$

where we set $c_{0}(M)=[1] \in H_{D R}^{0}(M)$. Let $H c_{q}(\Theta)$ be the harmonic part of $c_{q}(\Theta)$ in the Hodge decomposition. Since $M$ is Kähler, there exists a real $(q-1, q-1)$ form $f_{q}$ such that

$$
c_{q}(\Theta)-H c_{q}(\Theta)=\frac{\sqrt{-1}}{2 \pi} \partial \bar{\partial} f_{q}
$$

where $f_{q}$ is unique up to a $\partial \bar{\partial}$-closed form.

Definition 1.1. Let $\mathcal{F}_{q}: H^{0}\left(M, T^{1,0}(M)\right) \longrightarrow \mathbb{C}$. The $q$-th Bando-Futaki invariant is defined as

$$
\mathcal{F}_{q}(X)=\int_{M} \mathrm{~L}_{X} f_{q} \wedge \omega_{M}^{m+1-q}
$$

for $q=1, \cdots, m$.

Each $\mathcal{F}_{q}$ is well-defined on the Lie algebra of holomorphic vector fields and is independent of the choice of the Kähler form in the Kähler class. This property was proved by Bando 1 and can also be found in Futaki's book [6. In particular, $\mathcal{F}_{1}$ is known as the Futaki invariant. 
Theorem 1.1. Let $M$ be a hypersurface in $\mathbb{C P}^{n}$ defined by a homogeneous polynomial $F$ of degree $d$ with $d \leq n$. Let $X$ be a holomorphic vector field on $\mathbb{C P}^{n}$ such that

$$
X F=\kappa F
$$

for a constant $\kappa$. Then the $q$-th Bando-Futaki invariant is

$$
\mathcal{F}_{q}(X)=-(n+1-d)^{n-q} \frac{(d-1)(n+1)}{n} \sum_{j=0}^{q-1}(-d)^{j}(j+1)\left(\begin{array}{c}
n \\
q-j-1
\end{array}\right) \kappa .
$$

A hypersurface $M$ defined by the homogeneous polynomial $F=0$ of degree $d$ has positive first Chern class if $d \leq n$. In fact, $M$ has nonzero holomorphic vector fields.

A summary of the proof is as follows. The first step is to find the potential forms $f_{q}$ defined in (1.1) for $1 \leq q \leq n-1$. In order to do this, we compute the elementary symmetric polynomials by using the curvature tensors of the hypersurface in terms of local coordinates. Then we find the extra holomorphic forms so that the potentials $f_{q}$ can be expressed globally. The second step is to evaluate the Bando-Futaki invariants. We take the contraction of (1.1) with vector field $X$. Then we may write the resulting function as a $\bar{\partial}$-equation of $(q-1, q-1)$-forms. That is,

$$
\bar{\partial}\left[-q \tilde{P}^{q}(\nabla X, \Theta, \cdots, \Theta)+q \alpha_{q q} \theta \omega^{q-1}-i(X) \partial f_{q}\right]=0
$$

where $\theta$ is the Hamiltonian function of $\omega, \tilde{P}^{q}$ is the polarization of the $q$-th elementary symmetric polynomial, and $\alpha_{q q}$ is a constant. We denote that $\omega_{M}=(n+1-d) \omega$ and $\omega=\left.\omega_{F S}\right|_{M}$, where $\omega_{F S}$ is the Fubini-Study metric on $\mathbb{C P}^{n}$. By Hodge decomposition, we have

$$
-q \tilde{P}^{q}(\nabla X, \Theta, \cdots, \Theta)+q \alpha_{q q} \theta \omega^{q-1}-i(X) \partial f_{q}=\psi_{q}+\bar{\partial} \varphi_{q}
$$

where $\psi_{q}$ is the harmonic part and $\bar{\partial} \varphi_{q}$ is the exact part. In Lemma 2.5, we show that $\psi_{q}=C(q) \omega^{q-1}$ for some constant $C(q)$. In Lemma 2.6, we prove that

$$
q \int_{M} \tilde{P}^{q}(\nabla X, \Theta, \cdots, \Theta) \wedge \omega_{M}^{n-q}=0
$$

Then we reduce (1.2) to

$$
\begin{aligned}
\mathcal{F}_{q}(X) & =\int_{M} L_{X}\left(f_{q}\right) \wedge \omega_{M}^{n-q} \\
& =q \alpha_{q q}(n+1-d)^{n-q} \int_{M} \theta \omega^{n-1}-C(q)(n+1-q)^{n-q} \int_{M} \omega^{n-1} .
\end{aligned}
$$

By [12, Theorem 5.1], we have

$$
\int_{M} \theta \omega^{n-1}=\frac{\kappa}{n} \quad \text { and } \quad \int_{M} \omega^{n-1}=d
$$

Thus the Bando-Futaki invariant is

$$
\int_{M} L_{X} f_{q} \wedge \omega^{n-q}=(n+1-d)^{n-q}\left(\frac{q \alpha_{q q} \kappa}{n}-C(q) d\right) .
$$

This can be computed explicitly. We overcome two obstacles in our proof. The first obstacle is to show that the equality holds in (1.3). Note that it is not true that 
for any invariant polynomial $\varphi$,

$$
\int_{M} \varphi(\nabla X, \Theta, \cdots, \Theta) \wedge \omega^{n-q}=11
$$

For $q=1$, it is trivial to see that

$$
\int_{M} \tilde{P}^{1}(\nabla X) \wedge \omega_{M}^{n-1}=\int_{M} \operatorname{div}(X) \omega_{M}^{n-1}=\int_{M} L_{X}\left(\omega_{M}^{n-1}\right)=0 .
$$

However, (1.3) is an unexpected result for the polarization $\tilde{P}^{q}(\nabla X, \Theta, \cdots, \Theta)$ of the elementary polynomial for $2 \leq q \leq n-1$. Without this result, it would be very difficult to find the formula. The second obstacle is to compute $C(q)$ and to write $\varphi_{q}$ as a globally defined form.

Corollary $1.1(\mathrm{Lu},[12)$. Given the conditions of Theorem 1.1 and $q=1$, the first Bando-Futaki invariant is the same as the Futaki invariant given as

$$
\mathcal{F}_{1}(X)=-(n+1-d)^{n-1} \frac{(n+1)(d-1)}{n} \kappa .
$$

Our method can be applied, in principle, to the case of complete intersections and toric varieties. However, the computation is complicated using the current notation. Therefore our goal is to create an abstract setting from our current ideas before applying the invariant formula to more general cases.

In [6], the Futaki invariants (and Bando-Futaki invariants) are formularized as equivalent Chern numbers, which can be computed using the Atiyah-Bott localization formula. The method used in this paper is completely different from Atiyah and Bott's method and is an alternative way to compute the equivalent Chern numbers.

Remark 1.1. All Bando-Futaki invariants on a hypersurface vanish if the hypersurface is K-semistable.

Here we restate the definition [13:

Definition 1.2. We say $M$ is K-semistable if every holomorphic tangent vector field $X$ on $M$ satisfies

$$
\lim _{t \rightarrow 0} t \frac{d}{d t} M\left(\omega_{0}, \omega_{t}\right) \geq 0
$$

where $M\left(\omega_{0}, \omega_{t}\right)$ is the K-energy with respect to $\omega_{0}$ and $\omega_{t}$, and $\sigma(t)^{*} \omega_{0}=\omega_{t}$, where the one-parameter family of automorphisms $\sigma(t)$ is generated by the holomorphic vector field $X$.

In section 3, we study the holomorphic invariants that were introduced by Chen and Tian [3].

Definition 1.3. Let $M$ be an $n$-dimensional simply connected Kähler manifold with a Kähler form $\omega$ and $X$ be a holomorphic vector field over $M$. Since $M$ is simply connected, there exists a smooth function $\theta_{X}$ such that $i(X) \omega=\frac{\sqrt{-1}}{2 \pi} \bar{\partial} \theta_{X}$ 2

\footnotetext{
${ }^{1}$ If $\varphi(\nabla X, \Theta, \cdots, \Theta) \wedge \omega^{n-q}=\operatorname{div} X \cdot\left(c_{1}(\Theta)\right)^{n-1}$, then $\int_{M} \varphi(\nabla, \Theta, \cdots, \Theta) \wedge \omega^{n-q} \neq 0$.

${ }^{2}$ In order to keep the original definition, we use the equation $\theta_{X}=-\theta$, which is the opposite sign from the Hamiltonian function $\theta$ defined as above.
} 
For any positive integer $k$, define a new holomorphic invariant to be

$$
\begin{aligned}
F_{k}(X, \omega) & =(n-k) \int_{M} \theta_{X} \omega^{n}+(k+1) \int_{M} \Delta \theta_{X} \operatorname{Ric}(\omega)^{k} \wedge \omega^{n-k} \\
& -(n-k) \int_{M} \theta_{X} \operatorname{Ric}(\omega)^{k+1} \wedge \omega^{n-k-1} .
\end{aligned}
$$

Theorem 1.2. Let $M$ be a compact complex Kähler manifold with a Kähler form $[\omega] \in c_{1}(M)>0$. Then the Chen-Tian holomorphic invariants are the Futaki invariants:

$$
F_{k}(X, \omega)=(k+1) \int_{M} X\left(f_{\omega}\right) \omega^{n}
$$

where $f_{\omega}$ is a potential function such that $\operatorname{Ric}(\omega)-\omega=\frac{\sqrt{-1}}{2 \pi} \partial \bar{\partial} f_{\omega}$.

\section{BANDO-FUtAKI INVARIANTS}

2.1. Curvature tensor on hypersurfaces. The following setting is adopted from [15] and [13. Let $Z=\left[Z_{0}, \cdots, Z_{n}\right]$ be the homogeneous coordinate of $\mathbb{C P}^{n}$. Let $U_{0}=\left\{Z \in \mathbb{C P}^{n} \mid Z_{0} \neq 0\right\}$ and $z=\left(z_{1}, \cdots, z_{n}\right)$, where $z_{i}=Z_{i} / Z_{0}$ for $i=1, \cdots, n$. Without loss of generality, assume that we work on the coordinate chart $\left(U_{0}, z\right)$. Under this coordinate system, the Fubini-Study metric is given by

$$
\omega_{F S}=\frac{\sqrt{-1}}{2 \pi} \sum_{i, j=1}^{n} g_{i j} d z_{i} \wedge d \bar{z}_{j}=\frac{\sqrt{-1}}{2 \pi} \sum_{i, j=1}^{n}\left(\frac{\delta_{i j}}{1+|z|^{2}}-\frac{\bar{z}_{i} z_{j}}{\left(1+|z|^{2}\right)^{2}}\right) d z_{i} \wedge d \bar{z}_{j},
$$

where $|z|^{2}=\sum_{i=1}^{n}\left|z_{i}\right|^{2}$. Let $f$ be the defining polynomial of $M$ on $U_{0}$, where

$$
f(z)=F\left[1, \frac{Z_{1}}{Z_{0}}, \cdots, \frac{Z_{n}}{Z_{0}}\right]=\frac{1}{Z_{0}^{d}} F\left[Z_{0}, \cdots, Z_{n}\right] .
$$

By the implicit function theorem, there exists an open set $V \subseteq\left\{\left(z_{2}, \cdots, z_{n}\right) \in\right.$ $\left.\mathbb{C}^{n-1}\right\}$ of $U_{0}$ such that $z^{\prime}$ solves the equation $f\left(z_{1}, \cdots, z_{n}\right)=0$ whenever $\frac{\partial f}{\partial z_{1}}(z) \neq 0$. Let $z_{1}=z^{\prime}\left(z_{2}, \cdots, z_{n}\right)$. Therefore, $z_{1}$ is a holomorphic function on $V$. Under the coordinate system $\left(V,\left(z_{2}, \cdots, z_{n}\right)\right)$, let the restricted Kähler form be

$$
\omega=\left.\omega_{F S}\right|_{M}=\sum_{i, j=2}^{n} \frac{\sqrt{-1}}{2 \pi} \tilde{g}_{i \bar{j}} d z_{i} \wedge d \bar{z}_{j}
$$

where

$$
\tilde{g}_{i \bar{j}}=\frac{\delta_{i j}+a_{i} \bar{a}_{j}}{1+|z|^{2}}-\frac{\left(\bar{z}_{i}+\bar{z}_{1} a_{i}\right)\left(z_{j}+z_{1} \bar{a}_{j}\right)}{\left(1+|z|^{2}\right)^{2}} \quad \text { for } i, j=2, \cdots, n,
$$

where $a_{i}=\frac{\partial z_{1}}{\partial z_{i}}$ for $i=2, \cdots, n$. In order to compute the curvature tensor with respect to the metric $\tilde{g}_{i \bar{j}}$, it is critical to find the inverse matrix of $\tilde{g}_{i \bar{j}}$.

Lemma 2.1. Using the same notation as above, the inverse of $\tilde{g}_{i \bar{j}}$ is

$$
\begin{aligned}
\tilde{g}^{i \bar{j}}= & \frac{1}{\varrho}\left(\varrho\left(1+|z|^{2}\right) \delta_{j i}-a_{j} \bar{a}_{i}+\bar{z}_{j} z_{i}\left(1+|a|^{2}\right)\right) \\
& -\frac{1}{\varrho}\left(a_{j} z_{i}\left(\sum_{k=2}^{n} \bar{a}_{k} \bar{z}_{k}-\bar{z}_{1}\right)+\bar{z}_{j} \bar{a}_{i}\left(\sum_{k=2}^{n} a_{k} z_{k}-z_{1}\right)\right),
\end{aligned}
$$

where $\varrho=\frac{\sum_{k=0}^{n}\left|F_{k}\right|^{2}}{\left(1+|z|^{2}\right)\left|F_{1}\right|^{2}},|a|^{2}=\sum_{i=2}^{n}\left|a_{i}\right|^{2}$, and $F_{k}=\frac{\partial F}{\partial Z_{k}}$ for $k=0,1, \cdots, n$. 
Proof. Consider $\tilde{g}_{i \bar{j}}$ as a matrix $A_{i j}$ for $2 \leq i, j \leq n$. Since $\tilde{g}_{i \bar{j}}$ is a matrix of a linear combination of matrices $\delta_{i j}, a_{i} \bar{a}_{j}, \bar{z}_{i} z_{j}, a_{i} z_{j}$, and $\bar{z}_{i} \bar{a}_{j}$ pointwisely, its adjugate and inverse can be written as linear combinations of $\delta_{i j}, a_{i} \bar{a}_{j}, \bar{z}_{i} z_{j}, a_{i} z_{j}$, and $\bar{z}_{i} \bar{a}_{j}$ pointwisely. More precisely, let $A_{i j}=\left(\gamma_{1} \delta_{i j}+\gamma_{2} a_{i} \bar{a}_{j}+\gamma_{3} \bar{z}_{i} z_{j}+\gamma_{4} a_{i} z_{j}+\gamma_{5} \bar{z}_{i} \bar{a}_{j}\right)$, where

$$
\begin{aligned}
& \left(\gamma_{1}, \gamma_{2}, \gamma_{3}, \gamma_{4}, \gamma_{5}\right) \\
& =\left(\frac{1}{1+|z|^{2}}, \frac{1+|z|^{2}-\left|z_{1}\right|^{2}}{\left(1+|z|^{2}\right)^{2}},-\frac{1}{\left(1+|z|^{2}\right)^{2}},-\frac{\bar{z}_{1}}{\left(1+|z|^{2}\right)^{2}},-\frac{z_{1}}{\left(1+|z|^{2}\right)^{2}}\right) .
\end{aligned}
$$

By definition, the adjugate matrix is given by

$$
(\operatorname{adj} A)_{j i}=(-1)^{i+j} \sum_{\sigma \in S_{n-2}} \operatorname{sgn}(\sigma) A_{\sigma\left(i_{3}\right) j_{3}} \cdots A_{\sigma\left(i_{n}\right) j_{n}}
$$

where $S_{n-2}$ are all permutations of $\left\{i_{3}, \cdots, i_{n}\right\}=\{2, \cdots, n\}-\{i\}$ and $\left\{j_{3}, j_{4}\right.$, $\left.\cdots, j_{n}\right\}=\{2, \cdots, n\}-\{j\}$. There exist functions $\eta_{1}, \cdots, \eta_{5}$ such that

$$
(\operatorname{adj} A)_{j i}=\left(\eta_{1} \delta_{j i}+\eta_{2} a_{j} \bar{a}_{i}+\eta_{3} \bar{z}_{j} z_{i}+\eta_{4} a_{j} z_{i}+\eta_{5} \bar{z}_{j} \bar{a}_{i}\right) .
$$

Apply the following formulas given in [13]:

$$
\begin{array}{r}
\sum_{k=2}^{n} a_{k} z_{k}-z_{1}=\frac{F_{0}}{F_{1}}, \\
1+|a|^{2}+\left|\sum_{k=2}^{n} a_{k} z_{k}-z_{1}\right|^{2}=\frac{\sum_{k=0}^{n}\left|F_{k}\right|^{2}}{\left|F_{1}\right|^{2}}, \\
\operatorname{det} \tilde{g}_{i \bar{j}}=\frac{1}{\left(1+|z|^{2}\right)^{n}} \frac{\sum_{k=0}^{n}\left|F_{k}\right|^{2}}{\left|F_{1}\right|^{2}} .
\end{array}
$$

Then we can compute the coefficients $\eta_{1}, \cdots, \eta_{5}$ by solving the following linear equation system:

$$
\begin{aligned}
\tilde{g}_{i j} \tilde{g}^{k \bar{j}}= & \tilde{g}_{i \bar{j}} \frac{1}{\operatorname{det} \tilde{g}_{i \bar{j}}}(\operatorname{adj} A)_{j k} \\
= & \frac{1}{\operatorname{det} \tilde{g}_{i \bar{j}}}\left(\gamma_{1} \delta_{i j}+\gamma_{2} a_{i} \bar{a}_{j}+\gamma_{3} \bar{z}_{i} z_{j}+\gamma_{4} a_{i} z_{j}+\gamma_{5} \bar{z}_{i} \bar{a}_{j}\right) \\
& \times\left(\eta_{1} \delta_{j k}+\eta_{2} a_{j} \bar{a}_{k}+\eta_{3} \bar{z}_{j} z_{k}+\eta_{4} a_{j} z_{k}+\eta_{5} \bar{z}_{j} \bar{a}_{k}\right) \\
= & \delta_{i k} .
\end{aligned}
$$

Furthermore, we may rewrite (2.4) as

$$
\begin{aligned}
& \left(\gamma_{1}+\gamma_{2}|a|^{2}+\gamma_{4} \sum_{j=2}^{n} a_{j} z_{j}\right) \eta_{2}+\left(\gamma_{2} \sum_{j=2}^{n} \bar{a}_{j} \bar{z}_{j}+\gamma_{4} \sum_{j=2}^{n}\left|z_{j}\right|^{2}\right) \eta_{5}=-\gamma_{2} \eta_{1}, \\
& \left(\gamma_{1}+\gamma_{3} \sum_{j=2}^{n}\left|z_{j}\right|^{2}+\gamma_{5} \sum_{j=2}^{n} \bar{a}_{j} \bar{z}_{j}\right) \eta_{3}+\left(\gamma_{5}|a|^{2}+\gamma_{3} \sum_{j=2}^{n} a_{j} z_{j}\right) \eta_{4}=-\gamma_{3} \eta_{1}, \\
& \left(\gamma_{2} \sum_{j=2}^{n} \bar{a}_{j} \bar{z}_{j}+\gamma_{4} \sum_{j=2}^{n}\left|z_{j}\right|^{2}\right) \eta_{3}+\left(\gamma_{1}+\gamma_{2}|a|^{2}+\gamma_{4} \sum_{j=2}^{n} a_{j} z_{j}\right) \eta_{4}=-\gamma_{4} \eta_{1}, \\
& \left(\gamma_{3} \sum_{j=2}^{n} a_{j} z_{j}+\gamma_{5}|a|^{2}\right) \eta_{2}+\left(\gamma_{1}+\gamma_{3} \sum_{j=2}^{n}\left|z_{j}\right|^{2}+\gamma_{5} \sum_{j=2}^{n} \bar{a}_{j} \bar{z}_{j}\right) \eta_{5}=-\gamma_{5} \eta_{1},
\end{aligned}
$$


where $\eta_{1}=\operatorname{det} \tilde{g}_{i \bar{j}} \frac{1}{\gamma_{1}}=\left(1+|z|^{2}\right) \operatorname{det} \tilde{g}_{i \bar{j}}$. Hence by using (2.2) and (2.4), the solutions for (2.5) are

$$
\left(\eta_{2}, \eta_{3}, \eta_{4}, \eta_{5}\right)=\frac{1}{\left(1+|z|^{2}\right)^{n-1}}\left(-1,1+|a|^{2},-\left(\sum_{k=2}^{n} \bar{a}_{k} \bar{z}_{k}-\bar{z}_{1}\right),-\left(\sum_{k=2}^{n} a_{k} z_{k}-z_{1}\right)\right) .
$$

Then we may obtain

$$
\tilde{g}^{i \bar{j}}=\frac{1}{\operatorname{det} \tilde{g}_{i \bar{j}}}\left(\eta_{1} \delta_{j i}+\eta_{2} a_{j} \bar{a}_{i}+\eta_{3} \bar{z}_{j} z_{i}+\eta_{4} a_{j} z_{i}+\eta_{5} \bar{z}_{j} \bar{a}_{i}\right) .
$$

The following lemma is important for computing higher order Chern forms of the hypersurface $M$ and for evaluating the Bando-Futaki invariants.

Lemma 2.2. The curvature of the hypersurface is

$$
\sum_{i, j=2}^{n} R_{k i \bar{j}}^{\ell} d z_{i} \wedge d \bar{z}_{j}=\sum_{i, j=2}^{n}\left(\delta_{k \ell} \tilde{g}_{i \bar{j}}+\delta_{i \ell} \tilde{g}_{k \bar{j}}-\frac{1}{\rho} \frac{\partial a_{k}}{\partial z_{i}} \frac{\partial \bar{a}_{s}}{\partial \bar{z}_{j}} \tilde{g}^{\ell \bar{s}}\right) d z_{i} \wedge d \bar{z}_{j}
$$

for $2 \leq k, \ell \leq n$, where $\rho=\left(1+|z|^{2}\right) \frac{\sum_{k=0}^{n}\left|F_{k}\right|^{2}}{\left|F_{1}\right|^{2}}$.

Proof. Recall the curvature formula

$$
R_{k i \bar{j}}^{\ell}=-\frac{\partial^{2} \tilde{g}_{k \bar{s}}}{\partial z_{i} \partial \bar{z}_{j}} \tilde{g}^{\ell \bar{s}}+\frac{\partial \tilde{g}_{k \bar{q}}}{\partial z_{i}} \frac{\bar{\partial} \tilde{g}_{p \bar{s}}}{\partial \bar{z}_{j}} \tilde{g}^{p \bar{q}} \tilde{g}^{\ell \bar{s}}
$$

on Kähler manifolds. By using the metric $\tilde{g}_{i \bar{j}}$ defined in (2.1), we obtain

$$
\begin{aligned}
& \frac{\partial \tilde{g}_{k \bar{q}}}{\partial z_{i}}=-\frac{\bar{z}_{i}+\bar{z}_{1} a_{i}}{1+|z|^{2}} \tilde{g}_{k \bar{q}}-\frac{\bar{z}_{k}+\bar{z}_{1} a_{k}}{1+|z|^{2}} \tilde{g}_{i \bar{q}}+\frac{\partial a_{k}}{\partial z_{i}} \frac{\left(1+|z|^{2}-\left|z_{1}\right|^{2}\right) \bar{a}_{q}-\bar{z}_{1} z_{q}}{\left(1+|z|^{2}\right)^{2}} \\
& \frac{\partial \tilde{g}_{p \bar{s}}}{\partial \bar{z}_{j}}=-\frac{z_{j}+z_{1} \bar{a}_{j}}{1+|z|^{2}} \tilde{g}_{p \bar{s}}-\frac{z_{s}+z_{1} \bar{a}_{s}}{1+|z|^{2}} \tilde{g}_{p \bar{j}}+\frac{\partial \bar{a}_{s}}{\partial \bar{z}_{j}} \frac{\left(1+|z|^{2}-\left|z_{1}\right|^{2}\right) a_{p}-z_{1} \bar{z}_{p}}{\left(1+|z|^{2}\right)^{2}} .
\end{aligned}
$$

Use (2.8) to compute the second derivative

$$
\begin{aligned}
\frac{\partial^{2} \tilde{g}_{k \bar{s}}}{\partial z_{i} \partial \bar{z}_{j}}= & -\tilde{g}_{i j} \tilde{g}_{k \bar{s}}-\tilde{g}_{i \bar{s}} \tilde{g}_{k \bar{j}} \\
& +\frac{\left(z_{j}+z_{1} \bar{a}_{j}\right)\left(\bar{z}_{i}+\bar{z}_{1} a_{i}\right)}{\left(1+|z|^{2}\right)^{2}} \tilde{g}_{k \bar{s}}+\frac{\left(z_{j}+z_{1} \bar{a}_{j}\right)\left(\bar{z}_{k}+\bar{z}_{1} a_{k}\right)}{\left(1+|z|^{2}\right)^{2}} \tilde{g}_{i \bar{s}} \\
& +\frac{\left(z_{s}+z_{1} \bar{a}_{s}\right)\left(\bar{z}_{i}+\bar{z}_{1} a_{i}\right)}{\left(1+|z|^{2}\right)^{2}} \tilde{g}_{k \bar{j}}+\frac{\left(z_{s}+z_{1} \bar{a}_{s}\right)\left(\bar{z}_{k}+\bar{z}_{1} a_{k}\right)}{\left(1+|z|^{2}\right)^{2}} \tilde{g}_{i \bar{j}} \\
& -\frac{\left(z_{j}+z_{1} \bar{a}_{j}\right)}{1+|z|^{2}} \frac{\partial a_{k}}{\partial z_{i}} \frac{\left(1+|z|^{2}-\left|z_{1}\right|^{2}\right) \bar{a}_{s}-\bar{z}_{1} z_{s}}{\left(1+|z|^{2}\right)^{2}} \\
& -\frac{\left(z_{s}+z_{1} \bar{a}_{s}\right)}{1+|z|^{2}} \frac{\partial a_{k}}{\partial z_{i}} \frac{\left(1+|z|^{2}-|z|^{2}\right) \bar{a}_{j}-\bar{z}_{1} z_{j}}{\left(1+|z|^{2}\right)^{2}} \\
& +\frac{\partial a_{k}}{\partial z_{i}} \frac{\partial \bar{a}_{s}}{\partial \bar{z}_{j}} \frac{1+|z|^{2}-\left|z_{1}\right|^{2}}{\left(1+|z|^{2}\right)^{2}}+\frac{\partial \bar{a}_{s}}{\partial \bar{z}_{j}} \frac{a_{k} \bar{z}_{i}-a_{i} \bar{z}_{k}}{\left(1+|z|^{2}\right)^{2}} \\
& -2 \frac{\partial \bar{a}_{s}}{\partial \bar{z}_{j}}\left(\bar{z}_{i}+\bar{z}_{1} a_{i}\right) \frac{\left(1+|z|^{2}-\left|z_{1}\right|^{2}\right) a_{k}-z_{1} \bar{z}_{k}}{\left(1+|z|^{2}\right)^{3}} .
\end{aligned}
$$


By using (2.7) and (2.8) in (2.9), we obtain

$$
\begin{gathered}
\frac{\partial^{2} \tilde{g}_{k \bar{s}}}{\partial z_{i} \partial \bar{z}_{j}}=-\tilde{g}_{i \bar{j}} \tilde{g}_{k \bar{s}}-\tilde{g}_{i \bar{s}} \tilde{g}_{k \bar{j}}+\frac{\partial \tilde{g}_{k \bar{q}}}{\partial z_{i}} \frac{\bar{\partial} \tilde{g}_{p \bar{s}}}{\partial \bar{z}_{j}} \tilde{g}^{p \bar{q}}+\frac{\partial a_{k}}{\partial z_{i}} \frac{\partial \bar{a}_{s}}{\partial \bar{z}_{j}} \frac{1+|z|^{2}-\left|z_{1}\right|^{2}}{\left(1+|z|^{2}\right)^{2}} \\
-\frac{\partial a_{k}}{\partial z_{i}} \frac{\partial \bar{a}_{s}}{\partial \bar{z}_{j}}\left(\frac{\left(1+|z|^{2}-\left|z_{1}\right|^{2}\right) a_{p}-z_{1} \bar{z}_{p}}{\left(1+|z|^{2}\right)^{2}}\right)\left(\frac{\left(1+|z|^{2}-\left|z_{1}\right|^{2}\right) \bar{a}_{q}-\bar{z}_{1} z_{q}}{\left(1+|z|^{2}\right)^{2}}\right) \tilde{g}^{p \bar{q}} .
\end{gathered}
$$

In fact, we have

$$
\begin{array}{r}
\left(\frac{\left(1+|z|^{2}-\left|z_{1}\right|^{2}\right) a_{p}-z_{1} \bar{z}_{p}}{\left(1+|z|^{2}\right)^{2}}\right)\left(\frac{\left(1+|z|^{2}-\left|z_{1}\right|^{2}\right) \bar{a}_{q}-\bar{z}_{1} z_{q}}{\left(1+|z|^{2}\right)^{2}}\right) \tilde{g}^{p \bar{q}}-\frac{1+|z|^{2}-\left|z_{1}\right|^{2}}{\left(1+|z|^{2}\right)^{2}} \\
=-\frac{\left|F_{1}\right|^{2}}{\left(1+|z|^{2}\right) \sum_{k=0}^{n}\left|F_{k}\right|^{2}}=-\frac{1}{\rho} .
\end{array}
$$

By using (2.11) in (2.10), we get

$$
-\frac{\partial^{2} \tilde{g}_{k \bar{s}}}{\partial z_{i} \partial \bar{z}_{j}} \tilde{g}^{\ell \bar{s}}+\frac{\partial \tilde{g}_{k \bar{q}}}{\partial z_{i}} \frac{\bar{\partial} \tilde{g}_{p \bar{s}}}{\partial \bar{z}_{j}} \tilde{g}^{p \bar{q}} \tilde{g}^{\ell \bar{s}}=\delta_{k \ell} \tilde{g}_{i \bar{j}}+\delta_{i \ell} \tilde{g}_{k \bar{j}}-\frac{1}{\rho} \frac{\partial a_{k}}{\partial z_{i}} \frac{\partial \bar{a}_{s}}{\partial \bar{z}_{j}} \tilde{g}^{\ell \bar{s}} .
$$

The Ricci curvature of a hypersurface has been shown in [13, 15]. It also directly follows from Lemma 2.2 ,

Remark 2.1. Given the conditions as in Theorem 1.1, the Ricci curvature on the hypersurface is

where

$$
\operatorname{Ric}(\omega)=(n+1-d) \omega-\frac{\sqrt{-1}}{2 \pi} \partial \bar{\partial} \xi,
$$

$$
\xi=\log \left(\frac{\sum_{k=0}^{n}\left|F_{k}\right|^{2}}{\left(\sum_{k=0}^{n}\left|Z_{k}\right|^{2}\right)^{d-1}}\right) .
$$

Proof. The trace of the curvature form is the Ricci curvature. By using Lemma 2.2, we compute

$$
\begin{aligned}
\sum_{k=2}^{n} \sum_{i, j=2}^{n} R_{k i \bar{j}}^{k} d z_{i} \wedge d \bar{z}_{j} & =\sum_{k=2}^{n} \sum_{i, j=2}^{n}\left(\delta_{k k} \tilde{g}_{i \bar{j}}+\delta_{i k} \tilde{g}_{k \bar{j}}-\frac{1}{\rho} \frac{\partial a_{k}}{\partial z_{i}} \frac{\partial \bar{a}_{s}}{\partial \bar{z}_{j}} \tilde{g}^{k \bar{s}}\right) d z_{i} \wedge d \bar{z}_{j} \\
& =\sum_{i, j=2}^{n}\left(n \tilde{g}_{i \bar{j}}-\frac{1}{\rho} \sum_{k, s=2}^{n} \frac{\partial a_{k}}{\partial z_{i}} \frac{\partial \bar{a}_{s}}{\partial \bar{z}_{j}} \tilde{g}^{k \bar{s}}\right) d z_{i} \wedge d \bar{z}_{j}
\end{aligned}
$$

where

$$
\frac{1}{\rho} \frac{\sqrt{-1}}{2 \pi} \sum_{k, s=2}^{n} \frac{\partial a_{k}}{\partial z_{i}} \frac{\partial \bar{a}_{s}}{\partial \bar{z}_{j}} \tilde{g}^{k \bar{s}}=\partial_{i} \bar{\partial}_{j} \log \left(\frac{\sum_{k=0}^{n}\left|F_{k}\right|^{2}}{\left|F_{1}\right|^{2}}\right) .
$$

We can find an extra globally defined function such that

$$
\begin{aligned}
\frac{\sqrt{-1}}{2 \pi} \partial \bar{\partial} \log \left(\frac{\sum_{k=0}^{n}\left|F_{k}\right|^{2}}{\left|F_{1}\right|^{2}}\right) & =\frac{\sqrt{-1}}{2 \pi} \partial \bar{\partial} \log \left(\frac{\sum_{k=0}^{n}\left|F_{k}\right|^{2}}{\left(\sum_{k=0}^{n}\left|Z_{k}\right|^{2}\right)^{d-1}} \frac{\left(\sum_{k=0}^{n}\left|Z_{k}\right|^{2}\right)^{d-1}}{\left|F_{1}\right|^{2}}\right) \\
& =(d-1) \omega+\frac{\sqrt{-1}}{2 \pi} \partial \bar{\partial} \xi .
\end{aligned}
$$




\subsection{Chern forms.}

Lemma 2.3. Given the conditions in Theorem 1.1, the $q$-th Chern form on a hypersurface is

$$
c_{q}(\Theta)=\sum_{k=0}^{q} \alpha_{q k} \omega^{k} \wedge\left(\frac{\sqrt{-1}}{2 \pi} \partial \bar{\partial} \xi\right)^{q-k}
$$

where

$$
\begin{aligned}
\alpha_{00} & =1 \\
\alpha_{q q} & =\left(\begin{array}{c}
n+1 \\
q
\end{array}\right)-d \alpha_{(q-1)(q-1)}, \\
\alpha_{q(q-k)} & =-\left[d \alpha_{(q-1)(q-k-1)}+\alpha_{(q-1)(q-k)}\right] \quad \text { for } k=1, \cdots, q-1, \\
\alpha_{q 0} & =(-1)^{q},
\end{aligned}
$$

for $q=1, \cdots, n-1$.

Before we prove this lemma, we need several steps. Let $\Theta=\sum_{k, \ell=2}^{n} \Theta_{k}^{\ell} \frac{\partial}{\partial z_{\ell}} \otimes d z_{k}$ be the curvature tensor of $M$ such that

$$
\Theta_{k}^{\ell}=\frac{\sqrt{-1}}{2 \pi} \sum_{i, j=2}^{n} R_{k i \bar{j}}^{\ell} d z_{i} \wedge d \bar{z}_{j}
$$

represents a $(1,1)$-form-valued matrix for $2 \leq k, \ell \leq n$. In order to save some space, we denote the $(k, k)$-form-valued matrix $\sum_{i_{2}, i_{k}=2}^{n} \Theta_{i_{1}}^{i_{2}} \wedge \cdots \wedge \Theta_{i_{k}}^{i_{k+1}}$ by $\sum_{\text {is }}^{n} i_{2, \cdot, i_{k}=2} \Theta_{i_{1}}^{i_{2}} \cdots \Theta_{i_{k}}^{i_{k+1}}$. The $q$-th Chern polynomial defined in [9, pp. 402, 417]

$$
P^{q}(\Theta)=\frac{1}{q !} \sum_{\sigma \in S_{q}} \sum_{i_{1}, \cdots, i_{q}=2}^{n} \operatorname{sgn}(\sigma) \Theta_{i_{1}}^{i_{\sigma(1)}} \Theta_{i_{2}}^{i_{\sigma(2)}} \cdots \Theta_{i_{q}}^{i_{\sigma(q)}} .
$$

In Sublemma 2.1, we derive a formula to compute higher order Chern polynomials in terms of the lower order Chern polynomials. The idea is as follows. Let

$$
\phi_{j}=\sum_{i_{1}, \cdots, i_{j}=2}^{n} \Theta_{i_{1}}^{i_{2}} \Theta_{i_{2}}^{i_{3}} \cdots \Theta_{i_{j}}^{i_{1}}
$$

be a $(j, j)$-form corresponding to a cycle of order $j$. Every nontrivial permutation $\sigma \in S_{q}$ is a product of cyclic permutations which operate on disjoint indices: $\sigma=\sigma_{1} \sigma_{2} \cdots \sigma_{k}$. If $\sigma_{1}, \cdots, \sigma_{k}$ are cycles of length $j_{1}, \cdots, j_{k}$, respectively, then $\sum_{i_{1}, \cdots, i_{q}=2}^{n} \Theta_{i_{1}}^{i_{\sigma(1)}} \cdots \Theta_{i_{q}}^{i_{\sigma(q)}}=\phi_{j_{1}} \cdots \phi_{j_{k}}$. For each $1 \leq \ell \leq k$, let $\sigma=\sigma_{\ell} \tau_{\ell}$, where $\tau_{\ell}=\sigma_{1} \cdots \sigma_{\ell-1} \sigma_{\ell+1} \cdots \sigma_{k} \in S_{q-j_{\ell}}$. Since $\tau_{\ell}$ operates on $q-j_{\ell}$ many indices which are different from what $\sigma_{\ell}$ operates on, without loss of generality, assume that $\tau_{\ell}$ permutes $\left\{1, \cdots, q-j_{\ell}\right\}$ and $\sigma_{\ell}=\left(q-j_{\ell}+1 \cdots q\right)$. Then $\operatorname{sgn}\left(\tau_{\ell}\right) \sum_{i_{1}, \cdots, i_{q-j_{\ell}}=2}^{n} \Theta_{i_{1}}^{i_{\tau_{\ell}(1)}} \cdots \Theta_{i_{q-j_{\ell}}}^{i_{\tau_{\ell}\left(q-j_{\ell}\right)}}$ is one term in the expansion of $P^{q-j_{\ell}}(\Theta)$ as in (2.13). Therefore, we have

$$
\sum_{i_{1}, \cdots, i_{q}=2}^{n} \operatorname{sgn}(\sigma) \Theta_{i_{1}}^{i_{\sigma(1)}} \cdots \Theta_{i_{q}}^{i_{\sigma(q)}}=\operatorname{sgn}\left(\sigma_{\ell}\right) \operatorname{sgn}\left(\tau_{\ell}\right) \phi_{\ell} \sum_{i_{1}, \cdots, i_{q-j_{\ell}}=2}^{n} \Theta_{i_{1}}^{i_{\tau_{\ell}(1)}} \cdots \Theta_{i_{q-j_{\ell}}}^{i_{\tau_{\ell}\left(q-j_{\ell}\right)}},
$$

which belongs to one term in the expansion of $\operatorname{sgn}\left(\sigma_{\ell}\right) \phi_{j_{\ell}} P^{q-j_{\ell}}(\Theta)$. For every $\sigma \in$ $S_{q}, \sum_{i_{1}, \cdots, i_{q}=2}^{n} \operatorname{sgn}(\sigma) \Theta_{i_{1}}^{i_{\sigma(1)}} \cdots \Theta_{i_{q}}^{i_{\sigma(q)}}$ can be written as one term in the expansion of 
$\operatorname{sgn}\left(\sigma_{1}\right) \phi_{j_{1}} P^{q-j_{1}}(\Theta), \cdots$, or $\operatorname{sgn}\left(\sigma_{k}\right) \phi_{j_{k}} P^{q-j_{k}}(\Theta)$. Then we conclude that $P^{q}(\Theta)=$ $\sum_{j=1}^{q}(-1)^{j-1} a_{j} \phi_{j} P^{q-j}(\Theta)$ for some rational number $a_{j}$ for $j=1, \cdots, q$.

Sublemma 2.1. The q-th Chern polynomial can be written as

$$
P^{q}(\Theta)=\sum_{j=1}^{q}(-1)^{j-1} a_{j} \phi_{j} P^{q-j}(\Theta)=\frac{1}{q} \sum_{j=1}^{q}(-1)^{j-1} \phi_{j} P^{q-j}(\Theta)
$$

for $q=1, \cdots, n-1$. Let $P^{0}(\Theta)=1$ for convention.

Proof. Prove (2.14) by induction. For $q=1, P^{1}(\Theta)=\sum_{i=1}^{n} \Theta_{i}^{i}=\phi_{1}$. Assume that $P^{k}(\Theta)=\frac{1}{k} \sum_{j=1}^{k}(-1)^{j-1} \phi_{j} P^{k-j}(\Theta)$ holds for $k=2, \cdots, q-1$. Actually, there are $\left(\begin{array}{l}q \\ j\end{array}\right)(j-1)$ ! many cycles of order $j$ in $S_{q}$. For any positive integer numbers $j_{1}, \cdots, j_{k}$ such that $j_{1}+\cdots+j_{k}=q$, the coefficient for $\phi_{j_{1}} \cdots \phi_{j_{k}}$ on the left-hand side of (2.14) is

$$
\left\{\begin{array}{l}
\frac{1}{q !}\left(\begin{array}{c}
q \\
j_{1}
\end{array}\right)\left(j_{1}-1\right) !\left(\begin{array}{c}
q-j_{1} \\
j_{2}
\end{array}\right)\left(j_{2}-1\right) ! \cdots\left(\begin{array}{c}
j_{k} \\
j_{k}
\end{array}\right)\left(j_{k}-1\right) !, \quad \text { if } j_{t} \neq j_{s}, 1 \leq s, t \leq k ; \\
\frac{1}{\ell ! q !}\left(\begin{array}{c}
q \\
j_{1}
\end{array}\right)\left(j_{1}-1\right) !\left(\begin{array}{c}
q-j_{1} \\
j_{2}
\end{array}\right)\left(j_{2}-1\right) ! \cdots\left(\begin{array}{c}
j_{k} \\
j_{k}
\end{array}\right)\left(j_{k}-1\right) !, \text { if } \exists \ell \leq k, j_{1}=\cdots=j_{\ell},
\end{array}\right.
$$

and so on. Therefore, the coefficient for $\phi_{q}$ is $a_{q}=\frac{1}{q !}\left(\begin{array}{c}q \\ q\end{array}\right)(q-1) !=\frac{1}{q}$. For $q>$ $j>q / 2$, the coefficient for $\phi_{j} \phi_{q-j}$ on the left-hand side of (2.14) is $\frac{1}{q !}\left(\begin{array}{l}q \\ j\end{array}\right)(j-$ $1) !(q-j-1) !=\frac{1}{j(q-j)}$. On the other hand, since the term $\phi_{j} \phi_{q-j}$ only appears in the expansion of $\phi_{j} P^{q-j}(\Theta)$ and $\phi_{q-j} P^{j}(\Theta)$, by using the induction hypothesis, compare the coefficient of $\phi_{j} \phi_{q-j}$ on both sides of (2.14). That is,

$$
\frac{1}{j(q-j)}=\frac{a_{j}}{q-j}+\frac{a_{q-j}}{j} \text {. }
$$

If $q=2 k$ for some integer $k$, the coefficient of $\phi_{k} \phi_{k}$ is

$$
\frac{1}{2 ! q !}\left(\begin{array}{l}
q \\
k
\end{array}\right)(k-1) !(k-1) !=\frac{1}{k} a_{k}
$$

since $\phi_{k} \phi_{k}$ only appears in $\phi_{k} P^{k}(\Theta)$. Then $a_{k}=\frac{1}{2 k}=\frac{1}{q}$. For $j=q-2$, since $\phi_{q-2} \phi_{1} \phi_{1}$ only appears once in $\phi_{q-2} P^{2}(\Theta)$ and twice in $\phi_{1} P^{q-2}(\Theta)$, the coefficient is

$$
\frac{1}{2(q-2)}=\frac{a_{q-2}}{2}+\frac{a_{1}}{q-1}+\frac{a_{1}}{(q-1)(q-2)} .
$$

Furthermore, for $q-2>j>q-j-1>1$, the term $\phi_{j} \phi_{q-j-1} \phi_{1}$ appears twice in $\phi_{j} P^{q-j}(\Theta), \phi_{q-j-1} P^{j+1}(\Theta)$ and $\phi_{1} P^{q-1}(\Theta)$. The coefficient for $\phi_{j} \phi_{q-j-1} \phi_{1}$ is

$$
\frac{1}{j(q-j-1)}
$$

$$
\begin{aligned}
=\frac{a_{j}}{q-j}+\frac{a_{j}}{(q-j)(q-j-1)}+\frac{a_{q-j-1}}{j+1}+ & \frac{a_{q-j-1}}{(j+1) j}+\frac{a_{1}}{(q-1)(q-j-1)}+\frac{a_{1}}{(q-1) j} \\
& =\frac{a_{j}}{q-j-1}+\frac{a_{q-j-1}}{j}+\frac{a_{1}}{(q-j-1) j} .
\end{aligned}
$$


By using (2.15), (2.16), and (2.17), we obtain the relation of $a_{j}$

$$
\begin{aligned}
a_{q-1} & =t, \\
a_{q-j} & =\frac{-(j-1)+j(q-1) t}{q-j} \quad \text { for } j>q / 2, \\
a_{j} & =1-(q-1) t \quad \text { for } j<q / 2 .
\end{aligned}
$$

The term $\underbrace{\phi_{1} \cdots \phi_{1}}_{q}$ only appears in $\phi_{1} P^{q-1}(\Theta)$; the coefficient of it is

$$
\frac{1}{q ! q !}\left(\begin{array}{c}
q \\
1
\end{array}\right)\left(\begin{array}{c}
q-1 \\
1
\end{array}\right) \cdots\left(\begin{array}{l}
1 \\
1
\end{array}\right)=\frac{1}{q !}=\frac{a_{1}}{(q-1)(q-2) \cdots 1}
$$

We get $a_{1}=\frac{1}{q}$. By using (2.18), (2.19) and (2.20), we obtain $a_{j}=\frac{1}{q}$ for $j=$ $2, \cdots, q$.

Therefore, we only need to compute $\phi_{j}$ for $j=2, \cdots, q$ and use (2.14) to formulate $P^{q}(\Theta)$.

Sublemma 2.2. For $3 \leq j \leq q$, we have

$$
\begin{aligned}
& \sum_{i_{2}, \cdots, i_{j-1}=2}^{n} \Theta_{i_{1}}^{i_{2}} \cdots \Theta_{i_{j-1}}^{i_{j}}=\omega \sum_{i_{3}, \cdots, i_{j-1}=2}^{n} \Theta_{i_{1}}^{i_{3}} \Theta_{i_{3}}^{i_{4}} \cdots \Theta_{i_{j-1}}^{i_{j}} \\
& -\frac{\sqrt{-1}}{2 \pi} \frac{1}{\rho} \sum_{\alpha, \beta=2}^{n}\left(\frac{\partial a_{i_{1}}}{\partial z_{\alpha}} \frac{\partial \bar{a}_{s}}{\partial \bar{z}_{\beta}} \tilde{g}^{i_{j} \bar{s}} d z_{\alpha} \wedge d \bar{z}_{\beta}\right) \wedge\left(d \omega+\frac{\sqrt{-1}}{2 \pi} \partial \bar{\partial} \xi\right)^{j-2},
\end{aligned}
$$

where $\rho=\left(1+|z|^{2}\right) \frac{\sum_{k=0}^{n}\left|F_{k}\right|^{2}}{\left|F_{1}\right|^{2}}, \xi=\log \left(\frac{\sum_{k=0}^{n}\left|F_{k}\right|^{2}}{\left(\sum_{k=0}^{n}\left|Z_{k}\right|^{2}\right)^{d-1}}\right)$, and

$$
\Theta_{i}^{j}=\frac{\sqrt{-1}}{2 \pi} \sum_{\alpha, \beta=2}^{n} R_{i \alpha \bar{\beta}}^{j} d z_{\alpha} \wedge d \bar{z}_{\beta}
$$


Proof. Prove by induction. For $j=3$, use Lemma 2.2 and (2.12) to compute

$$
\begin{aligned}
& \sum_{i_{2}=2}^{n} \Theta_{i_{1}}^{i_{2}} \Theta_{i_{2}}^{i_{3}}=\left(\frac{\sqrt{-1}}{2 \pi}\right)^{2} \sum_{i_{2}, \alpha, \beta, \lambda, \eta, s, t=2}^{n}\left(\delta_{i_{1} i_{2}} \tilde{g}_{\alpha \bar{\beta}}+\delta_{\alpha i_{2}} \tilde{g}_{i_{1} \bar{\beta}}-\frac{1}{\rho} \frac{\partial a_{i_{1}}}{\partial z_{\alpha}} \frac{\partial \bar{a}_{s}}{\partial \bar{z}_{\beta}} \tilde{g}^{i_{2} \bar{s}}\right) \\
& \cdot\left(\delta_{i_{2} i_{3}} \tilde{g}_{\lambda \bar{\eta}}+\delta_{\lambda i_{3}} \tilde{g}_{i_{2} \bar{\eta}}-\frac{1}{\rho} \frac{\partial a_{i_{2}}}{\partial z_{\lambda}} \frac{\partial \bar{a}_{t}}{\partial \bar{z}_{\eta}} \tilde{g}^{i_{3} \bar{t}}\right) d z_{\alpha} \wedge d \bar{z}_{\beta} \wedge d z_{\lambda} \wedge d \bar{z}_{\eta} \\
& =\left(\frac{\sqrt{-1}}{2 \pi}\right)^{2} \sum_{i_{2}, \alpha, \beta, \lambda, \eta=2}^{n}\left[\delta_{i_{1} i_{3}} \tilde{g}_{\alpha \bar{\beta}} \tilde{g}_{\lambda \bar{\eta}}+\delta_{i_{3} \lambda} \tilde{g}_{\alpha \bar{\beta}} \tilde{g}_{i_{1} \bar{\eta}}\right. \\
& -\frac{1}{\rho} \sum_{t=2}^{n} \frac{\partial a_{i_{1}}}{\partial z_{\lambda}} \frac{\partial \bar{a}_{t}}{\partial \bar{z}_{\eta}} \tilde{g}^{i_{3} \bar{t}} \tilde{g}_{\alpha \bar{\beta}}-\frac{1}{\rho} \sum_{s=2}^{n} \frac{\partial a_{i_{1}}}{\partial z_{\alpha}} \frac{\partial \bar{a}_{s}}{\partial \bar{z}_{\beta}} \tilde{g}^{i_{3} \bar{s}} \tilde{g}_{\lambda \bar{\eta}} \\
& \left.+\frac{1}{\rho^{2}} \sum_{s, t=2}^{n}\left(\frac{\partial a_{i_{1}}}{\partial z_{\alpha}} \frac{\partial \bar{a}_{t}}{\partial \bar{z}_{\eta}} \tilde{g}^{i_{3} \bar{t}}\right)\left(\frac{\partial a_{i_{2}}}{\partial z_{\lambda}} \frac{\partial \bar{a}_{s}}{\partial \bar{z}_{\beta}} \tilde{g}^{i_{2} \bar{s}}\right)\right] d z_{\alpha} \wedge d \bar{z}_{\beta} \wedge d z_{\lambda} \wedge d \bar{z}_{\eta} \\
& =\omega \wedge \Theta_{i_{1}}^{i_{3}}-\frac{1}{\rho} \sum_{\alpha, \beta, s=2}^{n} \frac{\partial a_{i_{1}}}{\partial z_{\alpha}} \frac{\partial \bar{a}_{s}}{\partial \bar{z}_{\beta}} \tilde{g}^{i_{3} \bar{s}} d z_{\alpha} \wedge d \bar{z}_{\beta} \wedge \omega \\
& -\left(\frac{\sqrt{-1}}{2 \pi}\right)^{2} \sum_{\alpha, \beta, t, \lambda, \eta=2}^{n} \frac{1}{\rho} \frac{\partial a_{i_{1}}}{\partial z_{\alpha}} \frac{\partial \bar{a}_{t}}{\partial \bar{z}_{\eta}} \tilde{g}^{i_{3} \bar{t}}\left(\partial_{\lambda} \bar{\partial}_{\beta} \log \frac{\sum_{k=0}^{n}\left|F_{k}\right|^{2}}{\left|F_{1}\right|^{2}}\right) d z_{\alpha} \wedge d \bar{z}_{\eta} \wedge d z_{\lambda} \wedge d \bar{z}_{\beta} \\
& =\omega \wedge \Theta_{i_{1}}^{i_{3}}-\frac{\sqrt{-1}}{2 \pi} \frac{1}{\rho} \sum_{\alpha, \eta, t=2}^{n}\left(\frac{\partial a_{i_{1}}}{\partial z_{\alpha}} \frac{\partial \bar{a}_{t}}{\partial \bar{z}_{\eta}} \tilde{g}^{i_{3} \bar{t}} d z_{\alpha} \wedge d \bar{z}_{\eta}\right) \wedge\left(d \omega+\frac{\sqrt{-1}}{2 \pi} \partial \bar{\partial} \xi\right) .
\end{aligned}
$$

Suppose that the statement is true for $j-1$. By the induction hypothesis, we get

$$
\begin{aligned}
& \sum_{i_{2}, \cdots, i_{j-1}=2}^{n} \Theta_{i_{1}}^{i_{2}} \ldots \Theta_{i_{j-1}}^{i_{j}}=\left(\omega \wedge \sum_{i_{3}, \cdots, i_{j-1}=2}^{n} \Theta_{i_{1}}^{i_{3}} \Theta_{i_{3}}^{i_{4}} \cdots \Theta_{i_{j-2}}^{i_{j-1}}\right) \Theta_{i_{j-1}}^{i_{j}} \\
&-\frac{\sqrt{-1}}{2 \pi} \frac{1}{\rho} \sum_{i_{j-1}=2}^{n} \sum_{s, \alpha, \beta=2}^{n}\left(\frac{\partial a_{i_{1}}}{\partial z_{\alpha}} \frac{\partial \bar{a}_{s}}{\partial \bar{z}_{\beta}} \tilde{g}^{i_{j-1} \bar{s}} d z_{\alpha} \wedge d \bar{z}_{\beta}\right) \wedge\left(d \omega+\frac{\sqrt{-1}}{2 \pi} \partial \bar{\partial} \xi\right)^{j-3} \wedge \Theta_{i_{j-1}}^{i_{j}} \\
&=\omega \wedge \sum_{i_{3}, \cdots, i_{j-1}=2}^{n} \Theta_{i_{1}}^{i_{3}} \Theta_{i_{3}}^{i_{4}} \cdots \Theta_{i_{j-1}}^{i_{j}} \\
&-\frac{\sqrt{-1}}{2 \pi} \frac{1}{\rho} \sum_{\alpha, \beta=2}^{n}\left(\frac{\partial a_{i_{1}}}{\partial z_{\alpha}} \frac{\partial \bar{a}_{t}}{\partial \bar{z}_{\beta}} \tilde{g}^{i_{j} \bar{t}} d z_{\alpha} \wedge d \bar{z}_{\beta}\right) \wedge\left(d \omega+\frac{\sqrt{-1}}{2 \pi} \partial \bar{\partial} \xi\right)^{j-2},
\end{aligned}
$$

where

$$
\begin{aligned}
\sum_{i_{j-1}, \alpha, \beta, s=2}^{n}\left(\frac{\partial a_{i_{1}}}{\partial z_{\alpha}} \frac{\partial \bar{a}_{s}}{\partial \bar{z}_{\beta}} \tilde{g}^{i_{j-1} \bar{s}} d z_{\alpha} \wedge d \bar{z}_{\beta}\right) \wedge \Theta_{i_{j-1}}^{i_{j}} \\
=\sum_{\alpha, \beta, s=2}^{n}\left(\frac{\partial a_{i_{1}}}{\partial z_{\alpha}} \frac{\partial \bar{a}_{s}}{\partial \bar{z}_{\beta}} \tilde{g}^{i_{j} \bar{s}} d z_{\alpha} \wedge d \bar{z}_{\beta}\right) \wedge\left(d \omega+\frac{\sqrt{-1}}{2 \pi} \partial \bar{\partial} \xi\right)
\end{aligned}
$$

is part of (2.21). We omit the details of the proof. 
Theorem 2.1. With the curvature given in Lemma 2.2, the trace of the wedge product of $j$ copies of curvature tensors on the hypersurface $M$ is

$$
\sum_{i_{1}, \cdots, i_{j}=2}^{n} \Theta_{i_{1}}^{i_{2}} \cdots \Theta_{i_{j-1}}^{i_{j}} \Theta_{i_{j}}^{i_{1}}=(n+1) \omega^{j}-\left(d \omega+\frac{\sqrt{-1}}{2 \pi} \partial \bar{\partial} \xi\right)^{j}
$$

for $2 \leq j \leq q$

Proof. Prove by induction. For $j=2$, compute it directly from (2.21). That is,

$$
\begin{aligned}
\sum_{i_{1}, i_{2}=2}^{n} \Theta_{i_{1}}^{i_{2}} \Theta_{i_{2}}^{i_{1}}= & \omega \wedge\left((n+1-d) \omega-\frac{\sqrt{-1}}{2 \pi} \partial \bar{\partial} \xi\right) \\
& -\left((d-1) \omega+\frac{\sqrt{-1}}{2 \pi} \partial \bar{\partial} \xi\right)\left(d \omega+\frac{\sqrt{-1}}{2 \pi} \partial \bar{\partial} \xi\right) \\
= & (n+1) \omega^{2}-\left(d \omega+\frac{\sqrt{-1}}{2 \pi} \partial \bar{\partial} \xi\right)^{2} .
\end{aligned}
$$

Suppose the statement is true for $j-1$. By Sublemma2.2, the induction hypothesis and (2.22), we get

$$
\begin{aligned}
\sum_{i_{1}, i_{j}=2}^{n} & \left(\sum_{i_{2}, \cdots, i_{j-1}=2}^{n} \Theta_{i_{1}}^{i_{2}} \Theta_{i_{2}}^{i_{3}} \cdots \Theta_{i_{j-1}}^{i_{j}}\right) \Theta_{i_{j}}^{i_{1}} \\
=\omega & \wedge \sum_{i_{1}, i_{3}, \cdots, i_{j}=2}^{n} \Theta_{i_{1}}^{i_{3}} \Theta_{i_{3}}^{i_{4}} \cdots \Theta_{i_{j-1}}^{i_{j}} \Theta_{i_{j}}^{i_{1}} \\
& \quad-\frac{\sqrt{-1}}{2 \pi} \sum_{i_{1}, i_{j}, \alpha, \beta, s=2}^{n} \frac{\partial a_{i_{1}}}{\partial z_{\alpha}} \frac{\partial \bar{a}_{s}}{\partial \bar{z}_{\beta}} \tilde{g}^{i_{j} \bar{s}} d z_{\alpha} \wedge d \bar{z}_{\beta} \wedge\left(d \omega+\frac{\sqrt{-1}}{2 \pi} \partial \bar{\partial} \xi\right)^{j-2} \wedge \Theta_{i_{j}}^{i_{1}} \\
=\omega & \wedge\left[(n+1) \omega^{j-1}-\left(d \omega+\frac{\sqrt{-1}}{2 \pi} \partial \bar{\partial} \xi\right)^{j-1}\right] \\
& \quad-\left[(d-1) \omega+\frac{\sqrt{-1}}{2 \pi} \partial \bar{\partial} \xi\right] \wedge\left(d \omega+\frac{\sqrt{-1}}{2 \pi} \partial \bar{\partial} \xi\right)^{j-1} \\
= & (n+1) \omega^{j}-\left(d \omega+\frac{\sqrt{-1}}{2 \pi} \partial \bar{\partial} \xi\right)^{j} .
\end{aligned}
$$

Proof of Lemma 2.3. Prove by induction. For $q=1$,

$$
P^{1}(\Theta)=(n+1-d) \omega-\frac{\sqrt{-1}}{2 \pi} \partial \bar{\partial} \xi=\alpha_{10} \frac{\sqrt{-1}}{2 \pi} \partial \bar{\partial} \xi+\alpha_{11} \omega
$$

where $\alpha_{10}=(-1), \alpha_{11}=\left(\begin{array}{c}n+1 \\ 1\end{array}\right)-d \alpha_{00}$ and $\alpha_{00}=1$. Suppose that

$$
P^{i}(\Theta)=\sum_{k=0}^{i} \alpha_{i k} \omega^{k} \wedge\left(\frac{\sqrt{-1}}{2 \pi} \partial \bar{\partial} \xi\right)^{i-k}
$$


holds for $2 \leq i \leq q-1$. By Sublemma 2.1. Theorem 2.1 and the induction hypothesis, we have

$$
P^{q}(\Theta)=\frac{1}{q} \sum_{j=1}^{q}(-1)^{j-1} \phi_{j} P^{q-j}(\Theta)
$$

$$
=\frac{1}{q} \sum_{j=1}^{q}(-1)^{j-1}\left[(n+1) \omega^{j}-\left(d \omega+\frac{\sqrt{-1}}{2 \pi} \partial \bar{\partial} \xi\right)^{j}\right] \sum_{k=0}^{q-j} \alpha_{(q-j) k} \omega^{k} \wedge\left(\frac{\sqrt{-1}}{2 \pi} \partial \bar{\partial} \xi\right)^{q-j-k},
$$

where

$$
\begin{aligned}
\alpha_{(q-j) k} & = \begin{cases}-\left(d \alpha_{(q-j-1)(k-1)}+\alpha_{(q-j-1) k}\right), & \text { if } k<q-j ; \\
\left(\begin{array}{l}
n+1 \\
q-j
\end{array}\right)-d \alpha_{(q-j-1)(q-j-1)}, & \text { if } k=q-j .\end{cases} \\
& =(-1)^{q-j} \sum_{\ell=0}^{k}\left(\begin{array}{c}
q-j-\ell \\
k-\ell
\end{array}\right)(-1)^{\ell} d^{k-\ell}\left(\begin{array}{c}
n+1 \\
\ell
\end{array}\right)
\end{aligned}
$$

for $j=1, \cdots, q-1$. We rewrite $\left(\underline{2.23)}\right.$ as $P^{q}(\Theta)=\sum_{k=0}^{q} \alpha_{q k} \omega^{k}\left(\frac{\sqrt{-1}}{2 \pi} \partial \bar{\partial} \xi\right)^{q-k}$, where

$$
\begin{aligned}
\alpha_{q k}= & \frac{1}{q} \sum_{j=1}^{k}(-1)^{j-1}\left(n+1-d^{j}\right) \alpha_{(q-j)(k-j)} \\
& +\frac{1}{q} \sum_{i=1}^{q-k} \sum_{j=i}^{k+i}(-1)^{j} d^{j-i}\left(\begin{array}{c}
j \\
j-i
\end{array}\right) \alpha_{(q-j)(k-j+i)} .
\end{aligned}
$$

By using (2.24), we compute (2.25)

$$
\begin{aligned}
\alpha_{q k} & =\frac{n+1}{q}(-1)^{q-1} \sum_{j=1}^{k} \sum_{\ell=0}^{k-j}\left(\begin{array}{c}
q-j-\ell \\
k-j-\ell
\end{array}\right)(-1)^{\ell} d^{k-j-\ell}\left(\begin{array}{c}
n+1 \\
\ell
\end{array}\right) \\
& +\frac{(-1)^{q}}{q} \sum_{i=0}^{q-k} \sum_{j=i}^{k+i} \sum_{\ell=0}^{k-j+i}(-1)^{\ell} d^{k-\ell}\left(\begin{array}{c}
j \\
j-i
\end{array}\right)\left(\begin{array}{c}
q-j-\ell \\
k-j+i-\ell
\end{array}\right)\left(\begin{array}{c}
n+1 \\
\ell
\end{array}\right) \\
& -\frac{(-1)^{q}}{q} \sum_{\ell=0}^{k}\left(\begin{array}{c}
q-\ell \\
k-\ell
\end{array}\right)\left(\begin{array}{c}
n+1 \\
\ell
\end{array}\right) d^{k-\ell}(-1)^{\ell}
\end{aligned}
$$

In fact, we have

$$
\sum_{\ell=0}^{\lambda-1}(-1)^{\ell}\left(\begin{array}{c}
n+1 \\
\ell
\end{array}\right)=(-1)^{\lambda-1}\left(\begin{array}{c}
n \\
\lambda-1
\end{array}\right)
$$


We change the index in (2.26) and use (2.29) to get

$$
\begin{aligned}
\frac{n+1}{q}(-1)^{q-1} \sum_{j=1}^{k} \sum_{\ell=0}^{k-j}\left(\begin{array}{c}
q-j-\ell \\
k-j-\ell
\end{array}\right)(-1)^{\ell} d^{k-j-\ell}\left(\begin{array}{c}
n+1 \\
\ell
\end{array}\right) \\
=\frac{n+1}{q}(-1)^{q-1} \sum_{\lambda=1}^{k}\left(\begin{array}{c}
q-\lambda \\
k-\lambda
\end{array}\right) d^{k-\lambda} \sum_{\ell=0}^{\lambda-1}(-1)^{\ell}\left(\begin{array}{c}
n+1 \\
\ell
\end{array}\right) \\
=\frac{n+1}{q}(-1)^{q-1} \sum_{\lambda=1}^{k}\left(\begin{array}{c}
q-\lambda \\
k-\lambda
\end{array}\right) d^{k-\lambda}(-1)^{\lambda-1}\left(\begin{array}{c}
n \\
\lambda-1
\end{array}\right) \\
=\frac{1}{q}(-1)^{q} \sum_{\lambda=1}^{k}\left(\begin{array}{c}
q-\lambda \\
k-\lambda
\end{array}\right) d^{k-\lambda}(-1)^{\lambda} \lambda\left(\begin{array}{c}
n+1 \\
\lambda
\end{array}\right) .
\end{aligned}
$$

Change the summing order in (2.27) and get

$$
\begin{aligned}
& \frac{(-1)^{q}}{q} \sum_{i=0}^{q-k} \sum_{j=i}^{k+i} \sum_{\ell=0}^{k-j+i}(-1)^{\ell} d^{k-\ell}\left(\begin{array}{c}
j \\
j-i
\end{array}\right)\left(\begin{array}{c}
q-j-\ell \\
k-j+i-\ell
\end{array}\right)\left(\begin{array}{c}
n+1 \\
\ell
\end{array}\right) \\
= & \frac{(-1)^{q}}{q} \sum_{j=0}^{k}(-1)^{\ell} d^{k-\ell} \sum_{i=0}^{q-k} \sum_{\ell=0}^{j}\left(\begin{array}{c}
k+i-j \\
k-j
\end{array}\right)\left(\begin{array}{c}
q-k-i+j-\ell \\
j-\ell
\end{array}\right)\left(\begin{array}{c}
n+1 \\
\ell
\end{array}\right) \\
= & \frac{(-1)^{q}}{q} \sum_{\ell=0}^{k}(-1)^{\ell} d^{k-\ell} \sum_{j=\ell}^{k} \sum_{i=0}^{q-k}\left(\begin{array}{c}
k+i-j \\
k-j
\end{array}\right)\left(\begin{array}{c}
q-k-i+j-\ell \\
j-\ell
\end{array}\right)\left(\begin{array}{c}
n+1 \\
\ell
\end{array}\right) .
\end{aligned}
$$

In fact, we have

$$
\sum_{i=0}^{d}\left(\begin{array}{c}
s+i \\
s
\end{array}\right)=\left(\begin{array}{c}
s+d+1 \\
d+1
\end{array}\right) .
$$

Reassemble the following term:

$$
\begin{aligned}
\sum_{i=0}^{q-k}\left(\begin{array}{c}
k+i-j \\
k-j
\end{array}\right)\left(\begin{array}{c}
q-k-i+j-\ell \\
j-\ell
\end{array}\right) & =\sum_{i=0}^{q-k}\left(\begin{array}{c}
k+i-j \\
k-j
\end{array}\right) \\
+\sum_{s=1}^{q-k} \sum_{i=0}^{q-k-s}\left(\begin{array}{c}
k+i-j \\
k-j
\end{array}\right) & {\left[\left(\begin{array}{c}
s+j-\ell \\
j-\ell
\end{array}\right)-\left(\begin{array}{c}
s-1+j-\ell \\
j-\ell
\end{array}\right)\right] . }
\end{aligned}
$$

Then we may apply (2.32) to each assorted item in (2.33) to get

$$
\begin{aligned}
\sum_{i=0}^{q-k}\left(\begin{array}{c}
k+i-j \\
k-j
\end{array}\right)\left(\begin{array}{c}
q-k-i+j-\ell \\
j-\ell
\end{array}\right) \\
=\sum_{s=0}^{q-k}\left(\begin{array}{c}
k+s-(j-1) \\
k-(j-1)
\end{array}\right)\left(\begin{array}{c}
q-k-s+(j-1)-\ell \\
(j-1)-\ell
\end{array}\right) .
\end{aligned}
$$


If we repeat the procedure in (2.34) for $r$ times for $r=1, \cdots, j-\ell$, we get

$$
\begin{aligned}
\sum_{i=0}^{q-k}\left(\begin{array}{c}
k+i-j \\
k-j
\end{array}\right)\left(\begin{array}{c}
q-k-i+j-\ell \\
j-\ell
\end{array}\right) & =\sum_{i=0}^{q-k}\left(\begin{array}{c}
k+i-(j-r) \\
k-(j-r)
\end{array}\right)\left(\begin{array}{c}
q-k-i+(j-r)-\ell \\
(j-r)-\ell
\end{array}\right) .
\end{aligned}
$$

For each $j=r+\ell$, we have

$$
\sum_{i=0}^{q-k}\left(\begin{array}{c}
k+i-j \\
k-j
\end{array}\right)\left(\begin{array}{c}
q-k-i+j-\ell \\
j-\ell
\end{array}\right)=\sum_{i=0}^{q-k}\left(\begin{array}{c}
k+i-\ell \\
k-\ell
\end{array}\right)\left(\begin{array}{c}
q-k-i \\
0
\end{array}\right)=\left(\begin{array}{l}
q-\ell+1 \\
k-\ell+1
\end{array}\right) .
$$

Then we obtain

$$
\sum_{j=\ell}^{k} \sum_{i=0}^{q-k}\left(\begin{array}{c}
k+i-j \\
k-j
\end{array}\right)\left(\begin{array}{c}
q-k-i+j-\ell \\
j-\ell
\end{array}\right)=(k-\ell+1)\left(\begin{array}{l}
q-\ell+1 \\
k-\ell+1
\end{array}\right) .
$$

Use (2.35) in (2.31) to get

$$
\begin{array}{r}
\frac{(-1)^{q}}{q} \sum_{i=0}^{q-k} \sum_{j=i}^{k+i} \sum_{\ell=0}^{k-j+i}(-1)^{\ell} d^{k-\ell}\left(\begin{array}{c}
j \\
j-i
\end{array}\right)\left(\begin{array}{c}
q-j-\ell \\
k-j+i-\ell
\end{array}\right)\left(\begin{array}{c}
n+1 \\
\ell
\end{array}\right) \\
=\frac{(-1)^{q}}{q} \sum_{\ell=0}^{k}(-1)^{\ell} d^{k-\ell}(k-\ell+1)\left(\begin{array}{c}
q-\ell+1 \\
k-\ell+1
\end{array}\right)\left(\begin{array}{c}
n+1 \\
\ell
\end{array}\right) \\
=\frac{(-1)^{q}}{q} \sum_{\ell=0}^{k}(-1)^{\ell} d^{k-\ell}(q-\ell+1)\left(\begin{array}{c}
q-\ell \\
k-\ell
\end{array}\right)\left(\begin{array}{c}
n+1 \\
\ell
\end{array}\right) .
\end{array}
$$

By adding (2.30), (2.36) and (2.28), we can get the coefficient

$$
\begin{aligned}
\alpha_{q k}= & \frac{(-1)^{q}}{q} \sum_{\ell=1}^{k}\left(\begin{array}{c}
q-\ell \\
k-\ell
\end{array}\right) d^{k-\ell}(-1)^{\ell} \ell\left(\begin{array}{c}
n+1 \\
\ell
\end{array}\right) \\
& +\frac{(-1)^{q}}{q} d^{k}(q+1)\left(\begin{array}{c}
q \\
k
\end{array}\right)+\frac{(-1)^{q}}{q} \sum_{\ell=1}^{k}(-1)^{\ell} d^{k-\ell}(q-\ell+1)\left(\begin{array}{c}
q-\ell \\
k-\ell
\end{array}\right)\left(\begin{array}{c}
n+1 \\
\ell
\end{array}\right) \\
& -\frac{(-1)^{q}}{q} \sum_{\ell=0}^{k}(-1)^{\ell} d^{k-\ell}\left(\begin{array}{c}
q-\ell \\
k-\ell
\end{array}\right)\left(\begin{array}{c}
n+1 \\
\ell
\end{array}\right) \\
= & (-1)^{q} \sum_{\ell=0}^{k}(-1)^{\ell} d^{k-\ell}\left(\begin{array}{c}
q-\ell \\
k-\ell
\end{array}\right)\left(\begin{array}{c}
n+1 \\
\ell
\end{array}\right) .
\end{aligned}
$$

Comparing (2.37) and (2.24), we get

$$
\alpha_{q k}=\left\{\begin{array}{r}
\left(\begin{array}{c}
n+1 \\
q
\end{array}\right)-d \alpha_{(q-1)(q-1)}, \quad \text { if } k=q \\
-\left(d \alpha_{(q-1)(k-1)}+\alpha_{(q-1) k}\right), \quad \text { if } 0 \leq k<q
\end{array}\right.
$$


2.3. Computation of the Bando-Futaki invariants. In Lemma 2.3, we obtain the $q$-th Chern polynomial

$$
\begin{aligned}
c_{q}(\Theta) & =\sum_{k=0}^{q} \alpha_{q k} \omega^{k} \wedge\left(\frac{\sqrt{-1}}{2 \pi} \partial \bar{\partial} \xi\right)^{q-k} \\
& =\alpha_{q q} \omega^{q}+d \psi_{q}
\end{aligned}
$$

where $\psi_{q}=-\frac{\sqrt{-1}}{2 \pi} \partial \xi \wedge \sum_{k=0}^{q-1} \alpha_{q k} \omega^{k} \wedge\left(\frac{\sqrt{-1}}{2 \pi} \partial \bar{\partial} \xi\right)^{q-k-1}$. Then $c_{q}(\Theta)$ and $\alpha_{q q} \omega^{q}$ are in the same cohomology class in $H_{D R}^{2 q}(M)$. On any compact Kähler manifold, $\omega^{q}$ is harmonic for each $1 \leq q \leq n-1$. The Hodge theorem says that the harmonic representation of $c_{q}(\Theta)$ is unique on any compact Kähler manifold. That is, $H c_{q}(\Theta)=\alpha_{q q} \omega^{q}$. Actually, we have the Hodge decomposition

$$
c_{q}(\Theta)=\alpha_{q q} \omega^{q}+\frac{\sqrt{-1}}{2 \pi} \partial \bar{\partial} f_{q},
$$

where $f_{q}=\sum_{k=0}^{q-1} \alpha_{q k} \xi \omega^{q} \wedge\left(\frac{\sqrt{-1}}{2 \pi} \partial \bar{\partial} \xi\right)^{q-k-1}$ is globally defined. However, we will not directly compute the $q$-th Bando-Futaki invariant

$$
\mathcal{F}_{q}(X)=\int_{M} \mathcal{L}_{X} f_{q} \wedge \omega_{M}^{n-q-1},
$$

where $\omega_{M}=(n+1-d) \omega \in c_{1}(M)$ and $\omega=\left.\omega_{F S}\right|_{M}$.

First, take the contraction map on (2.38) with $X$ :

$$
i(X) c_{q}(\Theta)-i(X) \alpha_{q q} \omega_{q}=\frac{\sqrt{-1}}{2 \pi} \bar{\partial} i(X) \partial f_{q} .
$$

Let us compute each term in (2.39) separately. We take the contraction of $\omega^{q}$ with $X$ :

$$
i(X) \omega^{q}=q i(X)(\omega) \omega^{q-1}=q\left(-\frac{\sqrt{-1}}{2 \pi} \bar{\partial} \theta\right) \omega^{q-1},
$$

where $i(X) \omega=-\frac{\sqrt{-1}}{2 \pi} \bar{\partial} \theta$. More precisely, we can express a holomorphic vector field

$$
\tilde{X}=\sum_{i=0}^{n} \lambda_{i} Z_{i} \frac{\partial}{\partial Z_{i}}
$$

over $\mathbb{C P}^{n}$ with $\sum_{k=0}^{n} \lambda_{k}=0$. The restriction of $\tilde{X}$ on $M \cap V$ is given by

$$
\left.\tilde{X}\right|_{V}=X=\sum_{i=2}^{n}\left(\lambda_{i}-\lambda_{0}\right) z_{i}\left(a_{i} \frac{\partial}{\partial z_{1}}+\frac{\partial}{\partial z_{i}}\right) .
$$

Hence the Hamilton function can be expressed explicitly as

$$
\begin{aligned}
\theta & =-\tilde{X} \log \left(\sum_{k=0}^{n}\left|Z_{k}\right|^{2}\right)=-\frac{\sum_{k=0}^{n} \lambda_{k}\left|Z_{k}\right|^{2}}{\sum_{k=0}^{n}\left|Z_{k}\right|^{2}} \\
& =-\frac{\sum_{k=1}^{n} \lambda_{k}\left|z_{k}\right|^{2}+\lambda_{0}}{1+|z|^{2}}=-\frac{\sum_{k=1}^{n}\left(\lambda_{k}-\lambda_{0}\right)\left|z_{k}\right|^{2}}{1+|z|^{2}}-\lambda_{0} .
\end{aligned}
$$

Let $\tilde{P} \underbrace{(\Theta, \cdots, \Theta)}_{q}$ be the polarization of its elementary invariant polynomial $P^{q}(\Theta)$. 
Take the contraction of the curvature with $X$ :

$$
i(X) \Theta_{k}^{\ell}=i(X) \frac{\sqrt{-1}}{2 \pi} \sum_{i, j=2}^{n} R_{k i \bar{j}}^{\ell} d z_{i} \wedge d \bar{z}_{j}=\frac{\sqrt{-1}}{2 \pi} \sum_{i, j=2}^{n} X^{i} R_{k i \bar{j}}^{\ell} d \bar{z}_{j}=-\frac{\sqrt{-1}}{2 \pi} \bar{\partial} X_{k}^{\ell},
$$

where

$$
X_{k}^{\ell}=\frac{\partial X^{\ell}}{\partial z_{k}}+\sum_{i} X^{i} \Gamma_{i k}^{\ell}=-\sum_{j=2}^{n} \tilde{g}^{\bar{\ell}} \partial_{k} \bar{\partial}_{j} \theta
$$

for $2 \leq k, \ell \leq n$. Let

$$
\nabla X=\sum_{k, \ell} X_{k}^{\ell} d z_{k} \otimes \frac{\partial}{\partial z_{\ell}}=\sum_{k, \ell}\left(\frac{\partial X^{\ell}}{\partial z_{k}}+\sum_{i} X^{i} \Gamma_{k i}^{\ell}\right) d z_{k} \otimes \frac{\partial}{\partial z_{\ell}} .
$$

Then we can take the contraction map on the $q$-th Chern form

$$
i(X) c_{q}(\Theta)=q \tilde{P}^{q}(i(X) \Theta, \Theta, \cdots, \Theta)=-q \frac{\sqrt{-1}}{2 \pi} \bar{\partial} \tilde{P}^{q}(\nabla X, \Theta, \cdots, \Theta) .
$$

We may rewrite (2.39) as

$$
\bar{\partial}\left(-q \tilde{P}^{q}(\nabla X, \Theta, \cdots, \Theta)+q \alpha_{q q} \theta \omega^{q-1}-i(X) \partial f_{q, \Theta}\right)=0 .
$$

In fact, the space of harmonic forms $\mathcal{H}^{p, q}(M)$ and the Dolbeault cohomology $H_{\bar{\partial}}^{p, q}(M)$ groups are isomorphic. By the Hodge decomposition, the $\bar{\partial}$-closed form in (2.43) can be written as

$$
-q \tilde{P}^{q}(\nabla X, \Theta, \cdots, \Theta)+q \alpha_{q q} \theta \omega^{q-1}-i(X) \partial f_{q, \Theta}=\psi_{q}+\bar{\partial} \varphi_{q},
$$

where $\psi_{q}$ is the harmonic part of the left-hand side of (2.44) and $\varphi_{q}$ is a $(q-1, q-2)$ form. In Lemma 2.5, we will show that $\psi_{q}=C(q) \omega^{q-1}$ for some constant $C_{q}$. Instead of computing the Bando-Futaki invariants directly, we will compute the following:

$$
\begin{aligned}
& \int_{M} L_{X} f_{q} \wedge \omega_{M}^{n-q}=\int_{M}\left(\operatorname{di}(X) f_{q}+i(X) \partial f_{q}\right) \wedge \omega_{M}^{n-q} \\
& =\int_{M}\left(-q \tilde{P}^{q}(\nabla X, \Theta, \cdots, \Theta)+q \alpha_{q q} \theta \omega^{q-1}-C(q) \omega^{q-1}-\bar{\partial} \varphi_{q}\right) \wedge \omega_{M}^{n-q} .
\end{aligned}
$$

Finding $C(q)$ and showing $q \int_{M} \tilde{P}^{q}(\nabla X, \Theta, \cdots, \Theta) \wedge \omega_{M}^{n-q}=0$ are the next two steps to compute (2.45). In order to evaluate $C(q)$, it is necessary to express $\tilde{P}^{q}(\nabla X, \Theta, \cdots, \Theta)$ explicitly.

Lemma 2.4. The covariant derivative of the polarization of the elementary polynomial $P^{q}$ is given by

$$
\begin{aligned}
& q \tilde{P}^{q}(\nabla X, \Theta, \cdots, \Theta)=-\operatorname{div}(X) \gamma_{q 1}+\theta \gamma_{q 2} \\
& \quad-\sum_{j=2}^{q}(-1)^{j-1} \eta_{q-j} \wedge\left(\left(\omega^{j-2}+n \zeta_{j-2}\right) \wedge(\partial \bar{\partial} \theta)-\zeta_{j-2} \wedge(\partial \bar{\partial} \Delta \theta)\right),
\end{aligned}
$$


where

$$
\begin{aligned}
\gamma_{q 1} & =\sum_{k=0}^{q-1} \alpha_{q k}(q-k) \omega^{k} \wedge\left(\frac{\sqrt{-1}}{2 \pi} \partial \bar{\partial} \xi\right)^{q-1-k} \\
\gamma_{q 2} & =\sum_{k=0}^{q-1}\left((q-k)(n+1-d) \alpha_{q k}+(k+1) \alpha_{q(k+1)}\right) \omega^{k} \wedge\left(\frac{\sqrt{-1}}{2 \pi} \partial \bar{\partial} \xi\right)^{q-1-k} \\
\eta_{q-j} & =\sum_{k=0}^{q-j} \alpha_{(q-j) k} \frac{\sqrt{-1}}{2 \pi} \omega^{k} \wedge\left(\frac{\sqrt{-1}}{2 \pi} \partial \bar{\partial} \xi\right)^{q-j-k} \\
\zeta_{j-2} & =\sum_{k=0}^{j-2}\left(d \omega+\frac{\sqrt{-1}}{2 \pi} \partial \bar{\partial} \xi\right)^{j-2-k} \wedge \omega^{k} \\
\Delta \theta & =-\sum_{\alpha, \beta=2}^{n}
\end{aligned}
$$

Proof. According to Sublemma 2.1 we have

$$
\begin{aligned}
& q \tilde{P}^{q}(\nabla X, \Theta, \cdots, \Theta)=\frac{q}{q !} \sum_{\sigma \in S_{q}} \sum_{i_{1}, \cdots, i_{q}=2}^{n} \operatorname{sgn}(\sigma) X_{i_{1}}^{i_{\sigma(1)}} \Theta_{i_{2}}^{i_{\sigma(2)}} \cdots \Theta_{i_{2}}^{i_{\sigma(2)}} \\
& =\sum_{i_{1}=2}^{n} X_{i_{1}}^{i_{1}} P^{q-1}(\Theta)-\sum_{i_{1}, i_{2}}^{n} X_{i_{1}}^{i_{2}} \Theta_{i_{2}}^{i_{1}} P^{q-2}(\Theta)+\sum_{j=3}^{q}(-1)^{j-1} E_{j} P^{q-j}(\Theta),
\end{aligned}
$$

where

$$
E_{j}=\sum_{i_{1}, \cdots, i_{j}=2}^{n} X_{i_{1}}^{i_{2}} \Theta_{i_{2}}^{i_{3}} \cdots \Theta_{i_{j}}^{i_{1}}
$$

for $j=3, \cdots, q$. Let $E_{1}=\sum_{i=2}^{n} X_{i}^{i}$ and $E_{2}=\sum_{i, j=2}^{n} X_{i}^{j} \Theta_{i}^{j}$. In order to express $E_{j}$ as the differential forms that we have known, we need the following:

Sublemma 2.3. For $j=3, \cdots, q$, we have a regressive relation for $E_{j}$ :

$$
\begin{aligned}
E_{j} & =\omega \wedge E_{j-1}+\Phi \wedge\left(d \omega+\frac{\sqrt{-1}}{2 \pi} \partial \bar{\partial} \xi\right)^{j-2} \\
& =\omega^{j-2} \wedge E_{2}+\Phi \wedge \sum_{k=0}^{j-3}\left(d \omega+\frac{\sqrt{-1}}{2 \pi} \partial \bar{\partial} \xi\right)^{j-2-k} \omega^{k}
\end{aligned}
$$

where

$$
\Phi=-\frac{\sqrt{-1}}{2 \pi} \frac{1}{\rho} \sum_{k, \ell, p, q=2}^{n} X_{k}^{\ell} \frac{\partial a_{\ell}}{\partial z_{p}} \frac{\partial \bar{a}_{s}}{\partial \bar{z}_{q}} \tilde{g}^{k \bar{s}} d z_{p} \wedge d \bar{z}_{q} .
$$

Proof. Equation (2.48) will be proved by induction. For $j=3$, use Sublemma 2.2 to compute

$$
\begin{aligned}
E_{3}= & \sum_{i, j, k=2}^{3} X_{i}^{j} \Theta_{j}^{k} \Theta_{k}^{i}=\omega \wedge \sum_{i, j=2}^{n}\left(X_{i}^{j} \Theta_{j}^{i}\right) \\
& \quad-\frac{\sqrt{-1}}{2 \pi} \frac{1}{\rho} \sum_{\alpha, \beta=2}^{n} X_{i}^{j}\left(\frac{\partial a_{j}}{\partial z_{\alpha}} \frac{\partial \bar{a}_{s}}{\partial \bar{z}_{\beta}} \tilde{g}^{i \bar{s}} d z_{\alpha} \wedge d \bar{z}_{\beta}\right) \wedge\left(d \omega+\frac{\sqrt{-1}}{2 \pi} \partial \bar{\partial} \xi\right) \\
=\omega & \wedge E_{2}+\Phi \wedge\left(d \omega+\frac{\sqrt{-1}}{2 \pi} \partial \bar{\partial} \xi\right) .
\end{aligned}
$$


Assume that (2.48) holds for $j-1$. By Sublemma 2.2, the induction hypothesis, and (2.22), compute that

$$
\begin{gathered}
E_{j}=\sum_{i_{1}, \cdots, i_{j}=2}^{n} X_{i_{1}}^{i_{2}}\left(\Theta_{i_{2}}^{i_{3}} \cdots \Theta_{i_{j-1}}^{i_{j}}\right) \Theta_{i_{j}}^{i_{1}} \\
=\omega \sum_{i_{1}, i_{2}, i_{4} \cdots, i_{j}=2}^{n} X_{i_{1}}^{i_{2}} \Theta_{i_{2}}^{i_{4}} \Theta_{i_{4}}^{i_{5}} \cdots \Theta_{i_{j-1}}^{i_{j}} \Theta_{i_{j}}^{i_{1}} \\
-\frac{\sqrt{-1}}{2 \pi} \frac{1}{\rho} \sum_{i_{1}, i_{2}, i_{j}, \alpha, \beta=2}^{n} X_{i_{1}}^{i_{2}}\left(\frac{\partial a_{i_{2}}}{\partial z_{\alpha}} \frac{\partial \bar{a}_{s}}{\partial \bar{z}_{\beta}} \tilde{g}^{i_{j} \bar{s}} d z_{\alpha} \wedge d \bar{z}_{\beta}\right) \wedge\left(d \omega+\frac{\sqrt{-1}}{2 \pi} \partial \bar{\partial} \xi\right)^{j-3} \wedge \Theta_{i_{j}}^{i_{1}} \\
=\omega \wedge E_{j-1}-\frac{\sqrt{-1}}{2 \pi} \frac{1}{\rho} \sum_{i_{1}, i_{2}, i_{j}, \alpha, \beta=2}^{n} X_{i_{1}}^{i_{2}}\left(\frac{\partial a_{i_{2}}}{\partial z_{\alpha}} \frac{\partial \bar{a}_{s}}{\partial \bar{z}_{\beta}} \tilde{g}^{i_{1} \bar{s}} d z_{\alpha} \wedge d \bar{z}_{\beta}\right) \wedge\left(d \omega+\frac{\sqrt{-1}}{2 \pi} \partial \bar{\partial} \xi\right)^{j-2} \\
=\omega \wedge E_{j-1}+\Phi \wedge\left(d \omega+\frac{\sqrt{-1}}{2 \pi} \partial \bar{\partial} \xi\right)^{j-2} .
\end{gathered}
$$

Equation (2.49) follows directly from (2.48).

Before we start to compute $E_{j}$, we need to find $\Phi$.

Sublemma 2.4. We compute $\Phi$ explicitly as follows:

$$
\begin{aligned}
\Phi= & \operatorname{div}(X)\left((d-1) w+\frac{\sqrt{-1}}{2 \pi} \partial \bar{\partial} \xi\right)-n \frac{\sqrt{-1}}{2 \pi} \partial \bar{\partial} \theta+\frac{\sqrt{-1}}{2 \pi} \partial \bar{\partial} \Delta \theta \\
& -(n+1) \theta\left((d-1) w+\frac{\sqrt{-1}}{2 \pi} \partial \bar{\partial} \xi\right) .
\end{aligned}
$$

Proof. Using (2.42) in (2.50), $\Phi$ can be computed alternately by

$$
\Phi=\frac{\sqrt{-1}}{2 \pi} \frac{1}{\rho} \sum_{k, \ell, j, p, q=2}^{n} \tilde{g}^{\ell \bar{j}} \partial_{k} \bar{\partial}_{j} \theta \frac{\partial a_{\ell}}{\partial z_{p}} \frac{\partial \bar{a}_{s}}{\partial \bar{z}_{q}} \tilde{g}^{k \bar{s}} d z_{p} \wedge d \bar{z}_{q} .
$$

By using the definition of $\tilde{g}^{k \bar{s}}$ given in Lemma 2.1, we get

$$
\begin{aligned}
\varrho \frac{\partial a_{\ell}}{\partial z_{p}} \frac{\partial \bar{a}_{s}}{\partial \bar{z}_{q}} \tilde{g}^{k \bar{s}}=\frac{\sum_{i=0}^{n}\left|F_{i}\right|^{2}}{\left|F_{1}\right|^{2}} \frac{\partial a_{\ell}}{\partial z_{p}} \frac{\partial \bar{a}_{k}}{\partial \bar{z}_{q}}-\frac{\partial a_{\ell}}{\partial z_{p}} \bar{a}_{k} \sum_{s=2}^{n} \frac{\partial \bar{a}_{s}}{\partial \bar{z}_{q}} a_{s}+\left(1+|a|^{2}\right) \frac{\partial a_{\ell}}{\partial z_{p}} z_{k} \sum_{s=2}^{n} \frac{\partial \bar{a}_{s}}{\partial \bar{z}_{q}} \bar{z}_{s} \\
-\frac{\bar{F}_{0}}{\bar{F}_{1}} \frac{\partial a_{\ell}}{\partial z_{p}} z_{k} \sum_{s=2}^{n} \frac{\partial \bar{a}_{s}}{\partial \bar{z}_{q}} a_{s}-\frac{F_{0}}{F_{1}} \frac{\partial a_{\ell}}{\partial z_{p}} \overline{a_{k}} \sum_{s=2}^{n} \frac{\partial \bar{a}_{s}}{\partial \bar{z}_{q}} \bar{z}_{s} .
\end{aligned}
$$

By using the definition of $\theta$ in (2.41), we get

$$
\bar{\partial}_{j} \theta=-\frac{\left(\lambda_{j}-\lambda_{0}\right) z_{j}}{1+|z|^{2}}-\frac{\left(\lambda_{1}-\lambda_{0}\right) z_{1} \bar{a}_{j}}{1+|z|^{2}}-\left(\theta+\lambda_{0}\right) \frac{z_{j}+z_{1} \bar{a}_{j}}{1+|z|^{2}} .
$$


Then we compute

$$
\begin{aligned}
\partial_{k} \bar{\partial}_{j} \theta & =-\frac{\delta_{k j}\left(\lambda_{j}-\lambda_{0}\right)}{1+|z|^{2}}+\frac{\left(\lambda_{j}-\lambda_{0}\right) z_{j}\left(\bar{z}_{k}+\bar{z}_{1} a_{k}\right)}{\left(1+|z|^{2}\right)^{2}} \\
& -\frac{\left(\lambda_{1}-\lambda_{0}\right) a_{k} \bar{a}_{j}}{1+|z|^{2}}+\frac{\left(\lambda_{1}-\lambda_{0}\right) z_{1} \bar{a}_{j}\left(\bar{z}_{k}+\bar{z}_{1} a_{k}\right)}{\left(1+|z|^{2}\right)^{2}} \\
& +\frac{\left(\lambda_{k}-\lambda_{0}\right) \bar{z}_{k}}{1+|z|^{2}} \frac{z_{j}+z_{1} \bar{a}_{j}}{1+|z|^{2}}+\frac{\left(\lambda_{1}-\lambda_{0}\right) \bar{z}_{1} z_{k}}{1+|z|^{2}} \frac{z_{j}+z_{1} \bar{a}_{j}}{1+|z|^{2}} \\
& +2\left(\theta+\lambda_{0}\right) \frac{\left(\bar{z}_{k}+\bar{z}_{1} a_{k}\right)\left(z_{j}+z_{1} \bar{a}_{j}\right)}{\left(1+|z|^{2}\right)^{2}}-\left(\theta+\lambda_{0}\right) \frac{\delta_{k j}+a_{k} \bar{a}_{j}}{1+|z|^{2}} .
\end{aligned}
$$

A straightforward computation gives that

$$
\begin{aligned}
\tilde{g}^{\ell \bar{j}} \partial_{k} \bar{\partial}_{j} \theta & =-\delta_{\ell k}\left(\lambda_{\ell}-\lambda_{0}\right)+\frac{\left(\lambda_{\ell}-\lambda_{0}\right) z_{\ell}\left(\bar{z}_{k}+\bar{z}_{1} a_{k}\right)}{1+|z|^{2}}-\delta_{\ell k}\left(\theta+\lambda_{0}\right) \\
& +\bar{a}_{\ell} \frac{\left|F_{1}\right|^{2}}{\sum_{i=0}^{n}\left|F_{i}\right|^{2}} a_{k}\left(\lambda_{k}-\lambda_{0}\right)-\left(\lambda_{1}-\lambda_{0}\right) a_{k} \bar{a}_{\ell} \frac{\left|F_{1}\right|^{2}}{\sum_{i=0}^{n}\left|F_{i}\right|^{2}} \\
& -z_{\ell} \frac{\left|F_{1}\right|^{2}}{\sum_{i=0}^{n}\left|F_{i}\right|^{2}} \frac{\bar{F}_{0}}{\bar{F}_{1}} a_{k}\left(\left(\lambda_{1}-\lambda_{0}\right)-\left(\lambda_{k}-\lambda_{0}\right)\right) .
\end{aligned}
$$

Hence the Laplacian of $\theta$ is

$$
\begin{aligned}
\triangle \theta & =-\tilde{g}^{k \bar{j}} \partial_{k} \bar{\partial}_{j} \theta=-\left(\lambda_{0}+\frac{\left|F_{1}\right|^{2}}{\sum_{i=0}^{2}\left|F_{i}\right|^{2}} \sum_{k=1}^{n}\left(\lambda_{k}-\lambda_{0}\right)\left|a_{k}\right|^{2}-n \theta\right) \\
& =-\left(\frac{\sum_{k=0}^{n} \lambda_{k}\left|F_{k}\right|^{2}}{\sum_{i=0}^{n}\left|F_{i}\right|^{2}}-n \theta\right) .
\end{aligned}
$$

Denote $\operatorname{div}(X)=\sum_{i=2}^{n} X_{i}^{i}=\triangle \theta$.

Observe that

$$
\begin{aligned}
\partial_{p} \bar{\partial}_{q} \triangle \theta= & n \partial_{p} \bar{\partial}_{q} \theta-\frac{\left|F_{1}\right|^{2}}{\sum_{i=0}^{n}\left|F_{i}\right|^{2}} \sum_{k=2}^{n}\left(\lambda_{k}-\lambda_{0}\right) \frac{\partial a_{k}}{\partial z_{p}} \frac{\partial \bar{a}_{k}}{\partial \bar{z}_{q}} \\
& +\left(\frac{\left|F_{1}\right|^{2}}{\sum_{i=0}^{n}\left|F_{i}\right|^{2}}\right)^{2} \sum_{k=2}^{n}\left(\lambda_{k}-\lambda_{0}\right) \frac{\partial a_{k}}{\partial z_{p}} \bar{a}_{k}\left(\sum_{s=2}^{n} \frac{\partial \bar{a}_{s}}{\partial \bar{z}_{q}} a_{s}+\frac{F_{0}}{F_{1}} \sum_{s=2}^{n} \frac{\partial \bar{a}_{s}}{\partial \bar{z}_{q}} \bar{z}_{s}\right) \\
& +\left(\frac{\left|F_{1}\right|^{2}}{\sum_{i=0}^{n}\left|F_{i}\right|^{2}}\right)^{2} \sum_{k=2}^{n}\left(\lambda_{k}-\lambda_{0}\right) \frac{\partial \bar{a}_{k}}{\partial \bar{z}_{q}} a_{k}\left(\sum_{s=2}^{n} \frac{\partial a_{s}}{\partial z_{p}} \bar{a}_{s}+\frac{\bar{F}_{0}}{\bar{F}_{1}} \sum_{s=2}^{n} \frac{\partial a_{s}}{\partial z_{p}} z_{s}\right) \\
& -\sum_{k=1}^{n}\left(\lambda_{k}-\lambda_{0}\right)\left|a_{k}\right|^{2}\left(\frac{\left|F_{1}\right|^{2}}{\sum_{i=0}^{n}\left|F_{i}\right|^{2}}\right)^{3} \\
& \cdot\left(\sum_{s=2}^{n} \frac{\partial a_{s}}{\partial z_{p}} \bar{a}_{s}+\frac{\bar{F}_{0}}{\bar{F}_{1}} \sum_{s=2}^{n} \frac{\partial a_{s}}{\partial z_{p}} z_{s}\right)\left(\sum_{s=2}^{n} \frac{\partial \bar{a}_{s}}{\partial \bar{z}_{q}} a_{s}+\frac{F_{0}}{F_{1}} \sum_{s=2}^{n} \frac{\partial \bar{a}_{s}}{\partial \bar{z}_{q}} \bar{z}_{s}\right) \\
& +\sum_{k=1}^{n}\left(\lambda_{k}-\lambda_{0}\right)\left|a_{k}\right|^{2} \frac{\left|F_{1}\right|^{2}}{\sum_{i=0}^{n}\left|F_{i}\right|^{2}} \partial_{p} \bar{\partial}_{q} \log \left|\frac{\nabla F}{F_{1}}\right|^{2} .
\end{aligned}
$$


Then multiply (2.51) and (2.53) together to get

$$
\begin{aligned}
\frac{1}{\rho} \tilde{g}^{\ell \bar{j}} \partial_{k} \bar{\partial}_{j} \theta \frac{\partial a_{\ell}}{\partial z_{p}} \frac{\partial \bar{a}_{s}}{\partial \bar{z}_{q}} \tilde{g}^{k \bar{s}}= & \partial_{p} \bar{\partial}_{q} \triangle \theta-n \partial_{p} \bar{\partial}_{q} \theta-\left(\theta+\lambda_{0}\right) \partial_{p} \bar{\partial}_{q} \log \frac{\sum_{i=0}^{n}\left|F_{i}\right|^{2}}{\left|F_{1}\right|^{2}} \\
& -\left.\sum_{i=1}^{n}\left(\lambda_{i}-\lambda_{0}\right)\left|a_{i}\right|^{2} \frac{\left|F_{1}\right|^{2}}{\sum_{i=0}^{n}\left|F_{i}\right|^{2}}\right|^{2} \partial_{p} \bar{\partial}_{q} \log \frac{\sum_{i=0}^{n}\left|F_{i}\right|^{2}}{\left|F_{1}\right|^{2}} .
\end{aligned}
$$

Therefore, we obtain that

$$
\begin{aligned}
\Phi= & \frac{\sqrt{-1}}{2 \pi} \partial \bar{\partial} \triangle \theta-\frac{\sqrt{-1}}{2 \pi} n \partial \bar{\partial} \theta-\left(\theta+\lambda_{0}\right) \partial \bar{\partial} \log \frac{\sum_{i=0}^{2}\left|F_{i}\right|^{2}}{\left|F_{1}\right|^{2}} \\
& -\sum_{i=1}^{n}\left(\lambda_{i}-\lambda_{0}\right)\left|a_{i}\right|^{2} \frac{\left|F_{1}\right|^{2}}{\sum_{i=0}^{2}\left|F_{i}\right|^{2}} \partial \bar{\partial} \frac{\sum_{i=0}^{2}\left|F_{i}\right|^{2}}{\left|F_{1}\right|^{2}} \\
= & \frac{\sqrt{-1}}{2 \pi} \partial \bar{\partial} \triangle \theta-\frac{\sqrt{-1}}{2 \pi} n \partial \bar{\partial} \theta-(n+1) \theta\left((d-1) \omega+\frac{\sqrt{-1}}{2 \pi} \partial \bar{\partial} \xi\right) \\
& +\left(n \theta-\sum_{i=1}^{n}\left(\lambda_{i}-\lambda_{0}\right)\left|a_{i}\right|^{2} \frac{\left|F_{1}\right|^{2}}{\sum_{i=0}^{2}\left|F_{i}\right|^{2}}-\lambda_{0}\right)\left((d-1) \omega+\frac{\sqrt{-1}}{2 \pi} \partial \bar{\partial} \xi\right) .
\end{aligned}
$$

Then by using (2.54) and $\Delta \theta=\operatorname{div}(X)$, we get $\Phi$.

Sublemma 2.5. For $j=3, \cdots, q$, write $E_{j}$ explicitly as follows:

$$
\begin{aligned}
E_{j}= & \operatorname{div}(X) \psi_{j-1}-(n+1) \theta\left(\psi_{j-1}-\omega^{j-1}\right) \\
& -\left(\frac{\sqrt{-1}}{2 \pi} \partial \bar{\partial} \theta\right) \wedge\left(n \zeta_{j-2}+\omega^{j-2}\right)+\left(\frac{\sqrt{-1}}{2 \pi} \partial \bar{\partial} \Delta \theta\right) \wedge \zeta_{j-2},
\end{aligned}
$$

where $\zeta_{i}=\sum_{k=0}^{i}\left(d \omega+\frac{\sqrt{-1}}{2 \pi} \partial \bar{\partial} \xi\right)^{i-k} \omega^{k}$ and $\psi_{i}=\left(d \omega+\frac{\sqrt{-1}}{2 \pi} \partial \bar{\partial} \xi\right)^{i}$.

Proof. $E_{2}$ can be obtained directly from $\Phi$ :

$$
E_{2}=\sum_{i, j=2}^{n} X_{i}^{j} \Theta_{j}^{i}=\left(\operatorname{div}(X) \omega-\frac{\sqrt{-1}}{2 \pi} \partial \bar{\partial} \theta+\Phi\right) .
$$

Then by using (2.49) and Sublemma 2.4, we get

$$
\begin{aligned}
E_{j}= & E_{2} \wedge \omega^{j-2}+\Phi \wedge \sum_{k=0}^{j-3}\left(d \omega+\frac{\sqrt{-1}}{2 \pi} \partial \bar{\partial} \xi\right)^{j-2-k} \omega^{k} \\
= & \Phi \wedge \sum_{k=0}^{j-2}\left(d \omega+\frac{\sqrt{-1}}{2 \pi} \partial \bar{\partial} \xi\right)^{j-2-k} \omega^{k}+\operatorname{div}(X) \omega^{j-1}-\frac{\sqrt{-1}}{2 \pi} \partial \bar{\partial} \theta \wedge \omega^{j-2} \\
= & \operatorname{div}(X)\left(d \omega+\frac{\sqrt{-1}}{2 \pi} \partial \bar{\partial} \xi\right)^{j-1}-(n+1) \theta\left(\left(d \omega+\frac{\sqrt{-1}}{2 \pi} \partial \bar{\partial} \xi\right)^{j-1}-\omega^{j-1}\right) \\
& +\frac{\sqrt{-1}}{2 \pi} \partial \bar{\partial} \theta \wedge\left(n \sum_{k=0}^{j-2}\left(d \omega+\frac{\sqrt{-1}}{2 \pi} \partial \bar{\partial} \xi\right)^{j-2-k} \omega^{k}-\omega^{j-2}\right) \\
& +\frac{\sqrt{-1}}{2 \pi} \partial \bar{\partial} \triangle \theta \wedge \sum_{k=0}^{j-2}\left(d \omega+\frac{\sqrt{-1}}{2 \pi} \partial \bar{\partial} \xi\right)^{j-2-k} \omega^{k} .
\end{aligned}
$$


We return to the proof of Lemma 2.4. Denote $E_{1}=\sum_{i=2}^{n} X_{i}^{i}=\operatorname{div} X=\triangle \theta$. In fact, we can write down $E_{2}$ explicitly:

$$
\begin{aligned}
E_{2}= & \operatorname{div} X\left(d \omega+\frac{\sqrt{-1}}{2 \pi} \partial \bar{\partial} \xi\right)-(n+1) \frac{\sqrt{-1}}{2 \pi} \partial \bar{\partial} \theta \\
& +\frac{\sqrt{-1}}{2 \pi} \partial \bar{\partial} \triangle \theta-(n+1) \theta\left((d-1) \omega+\frac{\sqrt{-1}}{2 \pi} \partial \bar{\partial} \xi\right) .
\end{aligned}
$$

Then we can use Sublemma 2.5 to compute $\sum_{j=1}^{q}(-1)^{j-1} E_{j} P^{q-j}(\Theta)$. The coefficient for $\operatorname{div}(X)$ in (2.47) is given by

$$
\begin{aligned}
& \sum_{j=1}^{q}(-1)^{j-1} \psi_{j-1} \sum_{k=0}^{q-j} \alpha_{(q-j) k} \omega^{k}\left(\frac{\sqrt{-1}}{2 \pi} \partial \bar{\partial} \xi\right)^{q-j-k} \\
= & \sum_{j=1}^{q}(-1)^{j-1}\left(d \omega+\frac{\sqrt{-1}}{2 \pi} \partial \bar{\partial} \xi\right)^{j-1} \sum_{k=0}^{q-j} \alpha_{(q-j) k} \omega^{k}\left(\frac{\sqrt{-1}}{2 \pi} \partial \bar{\partial} \xi\right)^{q-j-k} \\
= & \sum_{t=1}^{q} \sum_{\ell=0}^{t-1} \sum_{j=t-\ell}^{q-\ell} \alpha_{(q-j)(q-j-\ell)}(-1)^{j-1} d^{j+\ell-t}\left(\begin{array}{c}
j-1 \\
j+\ell-t
\end{array}\right) \omega^{q-t}\left(\frac{\sqrt{-1}}{2 \pi} \partial \bar{\partial} \xi\right)^{t-1} .
\end{aligned}
$$

We derive several formulas before we perform further computations. For each fixed index $j \geq t$, use (2.24) to get

$$
\begin{aligned}
& \text {.57) } \sum_{\ell=0}^{t-1} \alpha_{(q-j)(q-j-\ell)}(-1)^{j-1} d^{j+\ell-t}\left(\begin{array}{c}
j-1 \\
j+\ell-t
\end{array}\right) \\
& =\sum_{\ell=1}^{t-1}(-1)\left(d \alpha_{(q-j-1)(q-j-\ell-1)}+\alpha_{(q-j-1)(q-j-\ell)}\right)(-1)^{j-1} d^{j+\ell-t}\left(\begin{array}{c}
j-1 \\
j+\ell-t
\end{array}\right) \\
& +(-1)^{j-1} d^{j-t}\left(\left(\begin{array}{c}
n+1 \\
q-j
\end{array}\right)-d \alpha_{(q-j-1)(q-j-1)}\right)\left(\begin{array}{c}
j-1 \\
j-t
\end{array}\right) .
\end{aligned}
$$

Rewrite (2.57) to get

$$
\begin{aligned}
& \sum_{\ell=0}^{t-1} \alpha_{(q-j)(q-j-\ell)}(-1)^{j-1} d^{j+\ell-t}\left(\begin{array}{c}
j-1 \\
j+\ell-t
\end{array}\right) \\
& =\sum_{\ell=1}^{t-2} \alpha_{(q-j-1)(q-j-\ell-1)}(-1)^{j} d^{j+1+\ell-t}\left[\left(\begin{array}{c}
j \\
j+1+\ell-t
\end{array}\right)-\left(\begin{array}{c}
j-1 \\
j+\ell+1-t
\end{array}\right)\right] \\
& +\alpha_{(q-j-1)(q-j-t)}(-d)^{j}+\alpha_{(q-j-1)(q-j-1)}(-1)^{j} d^{j+1-t}\left(\begin{array}{c}
j-1 \\
j+1-t
\end{array}\right) \\
& +\sum_{\ell=1}^{t-2} \alpha_{(q-j-1)(q-j-\ell-1)}(-1)^{j} d^{j+\ell+1-t}\left(\begin{array}{c}
j-1 \\
j+\ell+1-t
\end{array}\right) \\
& +(-1)^{j-1} d^{j-t}\left(\left(\begin{array}{c}
n+1 \\
q-j
\end{array}\right)-d \alpha_{(q-j-1)(q-j-1)}\right)\left(\begin{array}{l}
j-1 \\
j-t
\end{array}\right) .
\end{aligned}
$$


Cancelling out the same terms in (2.58), we obtain

$$
\begin{aligned}
& \sum_{\ell=0}^{t-1} \alpha_{(q-j)(q-j-\ell)}(-1)^{j-1} d^{j+\ell-t}\left(\begin{array}{c}
j-1 \\
j+\ell-t
\end{array}\right) \\
& \quad=\sum_{\ell=0}^{t-1} \alpha_{(q-j-1)(q-j-\ell-1)}(-1)^{j} d^{j+1+\ell-t}\left(\begin{array}{c}
j \\
j+1+\ell-t
\end{array}\right) \\
& +(-1)^{j-1} d^{j-t}\left(\begin{array}{c}
n+1 \\
q-j
\end{array}\right)\left(\begin{array}{c}
j-1 \\
j-t
\end{array}\right) \quad \text { for } j \geq t .
\end{aligned}
$$

Similarly to (2.59), we have

$$
\begin{aligned}
& \sum_{\ell=t-j}^{t-1} \alpha_{(q-j)(q-j-\ell)}(-1)^{j-1} d^{j+\ell-t}\left(\begin{array}{c}
j-1 \\
j+\ell-t
\end{array}\right) \\
& \quad=\sum_{\ell=t-j-1}^{t-1} \alpha_{(q-j-1)(q-j-\ell-1)}(-1)^{j} d^{j+1+\ell-t}\left(\begin{array}{c}
j \\
j+1+\ell-t
\end{array}\right) \text { for } j<t .
\end{aligned}
$$

For each $1 \leq s \leq q-2 t+1$, we divide the following summation into two parts:

$$
\begin{aligned}
\sum_{\ell=0}^{t-1} \sum_{j=t-\ell}^{q-\ell} \alpha_{(q-j)(q-j-\ell)}(-1)^{j-1} d^{j+\ell-t}\left(\begin{array}{c}
j-1 \\
j+\ell-t
\end{array}\right) & \\
= & \sum_{\ell=0}^{t-1} \sum_{j=t+s}^{q-\ell} \alpha_{(q-j)(q-j-\ell)}(-1)^{j-1} d^{j+\ell-t}\left(\begin{array}{c}
j-1 \\
j+\ell-t
\end{array}\right) \\
& +\sum_{j=1}^{t+s-1} \sum_{\ell=\max \{0, t-j\}}^{t-1} \alpha_{(q-j)(q-j-\ell)}(-1)^{j-1} d^{j+\ell-t}\left(\begin{array}{c}
j-1 \\
j+\ell-t
\end{array}\right) .
\end{aligned}
$$

For $s=1$ in the second part of (2.61), by (2.60) for each fixed $j$, each summation in $\ell$ is equal to each other. Then we use $j=t$ as the representation.

That is,

$$
\begin{aligned}
& \sum_{j=1}^{t} \sum_{\ell=t-j}^{t-1} \alpha_{(q-j)(q-j-\ell)}(-1)^{j-1} d^{j+\ell-t}\left(\begin{array}{c}
j-1 \\
j+\ell-t
\end{array}\right) \\
&=t \sum_{\ell=0}^{t-1} \alpha_{(q-t)(q-t-\ell)}(-1)^{t-1} d^{\ell}\left(\begin{array}{c}
t-1 \\
\ell
\end{array}\right) .
\end{aligned}
$$

By (2.62), we have

$$
\begin{aligned}
\sum_{j=1}^{t+s-1} \sum_{\ell=\max \{0, t-j\}}^{t-1} \alpha_{(q-j)(q-j-\ell)}(-1)^{j-1} d^{j+\ell-t}\left(\begin{array}{c}
j-1 \\
j+\ell-t
\end{array}\right) & \\
=t \sum_{\ell=0}^{t-1} \alpha_{(q-t)(q-t-\ell)}(-1)^{t-1} d^{\ell}\left(\begin{array}{c}
t-1 \\
\ell
\end{array}\right) & \\
& +\sum_{j=t+1}^{t+s-1} \sum_{\ell=0}^{t-1} \alpha_{(q-j)(q-j-\ell)}(-1)^{j-1} d^{j+\ell-t}\left(\begin{array}{c}
j-1 \\
j+\ell-t
\end{array}\right) .
\end{aligned}
$$


By using (2.59) in (2.63), we obtain

$$
\begin{gathered}
\sum_{j=1}^{t+s-1} \sum_{\ell=\max \{0, t-j\}}^{t-1} \alpha_{(q-j)(q-j-\ell)}(-1)^{j-1} d^{j+\ell-t}\left(\begin{array}{c}
j-1 \\
j+\ell-t
\end{array}\right) \\
=t \sum_{\ell=0}^{t-1} \alpha_{(q-t-1)(q-t-1-\ell)}(-1)^{t} d^{\ell+1}\left(\begin{array}{c}
t \\
\ell+1
\end{array}\right)+t(-1)^{t-1}\left(\begin{array}{c}
n+1 \\
q-t
\end{array}\right) \\
+\sum_{j=t+1}^{t+s-1} \sum_{\ell=0}^{t-1} \alpha_{(q-j)(q-j-\ell)}(-1)^{j-1} d^{j+\ell-t}\left(\begin{array}{c}
j-1 \\
j+\ell-t
\end{array}\right) \\
=(t+1) \sum_{\ell=0}^{t-1} \alpha_{(q-(t+1))(q-(t+1)-\ell)}(-1)^{t} d^{\ell+1}\left(\begin{array}{c}
t \\
\ell+1
\end{array}\right)+t(-1)^{t-1}\left(\begin{array}{c}
n+1 \\
q-t
\end{array}\right) \\
+\sum_{j=t+2}^{t+s-1} \sum_{\ell=0}^{t-1} \alpha_{(q-j)(q-j-\ell)}(-1)^{j-1} d^{j+\ell-t}\left(\begin{array}{c}
j-1 \\
j+\ell-t
\end{array}\right) .
\end{gathered}
$$

Repeat the procedure in (2.64) and put it back into (2.61) to get

$$
\begin{aligned}
& \sum_{\ell=0}^{t-1} \sum_{j=t-\ell}^{q-\ell} \alpha_{(q-j)(q-j-\ell)}(-1)^{j-1} d^{j+\ell-t}\left(\begin{array}{c}
j-1 \\
j+\ell-t
\end{array}\right) \\
& =\sum_{\ell=0}^{t-1} \sum_{j=t+s}^{q-\ell} \alpha_{(q-j)(q-j-\ell)}(-1)^{j-1} d^{j+\ell-t}\left(\begin{array}{c}
j-1 \\
j+\ell-t
\end{array}\right) \\
& +(t+s-1) \sum_{\ell=0}^{t-1} \alpha_{(q-(t+s-1))(q-(t+s-1)-\ell)}(-1)^{t-s-2} d^{s+\ell-1}\left(\begin{array}{c}
t-s-2 \\
s+\ell-1
\end{array}\right) \\
& +\sum_{j=t}^{t+s-2} j(-1)^{j-1} d^{j-t}\left(\begin{array}{c}
n+1 \\
q-j
\end{array}\right)\left(\begin{array}{c}
j-1 \\
j-t
\end{array}\right) \quad \text { for } 1 \leq s \leq q-2 t+1 .
\end{aligned}
$$

For $s=q-2 t+1$ in (2.65), we change the indexes in the first term on the right-hand side of it. That is,

$$
\begin{aligned}
& \sum_{\ell=0}^{t-1} \sum_{j=t+s}^{q-\ell} \alpha_{(q-j)(q-j-\ell)}(-1)^{j-1} d^{j+\ell-t}\left(\begin{array}{c}
j-1 \\
j+\ell-t
\end{array}\right) \\
& =\sum_{\ell=0}^{t-1} \sum_{j=q-t+1}^{q-\ell} \alpha_{(q-j)(q-j-\ell)}(-1)^{j-1} d^{j+\ell-t}\left(\begin{array}{c}
j-1 \\
j+\ell-t
\end{array}\right) \\
& =\sum_{j=0}^{t-1} \sum_{\ell=0}^{t-1-j} \alpha_{(t-1-j)(t-1-j-\ell)}(-1)^{q-t+j} d^{q-2 t+j+\ell+1}\left(\begin{array}{c}
q-t+j \\
q-2 t+j+\ell+1
\end{array}\right) .
\end{aligned}
$$


With the same procedure as we do in (2.59), we have

$$
\begin{array}{r}
\sum_{\ell=0}^{t-1-\lambda} \alpha_{(t-1-\lambda)(t-1-\lambda-\ell)}(-1)^{q-t+\lambda} d^{q-2 t+\lambda+\ell+1}\left(\begin{array}{c}
q-t+\lambda \\
q-2 t+\lambda+\ell+1
\end{array}\right) \\
=\sum_{\ell=0}^{t-2-\lambda} \alpha_{(t-2-\lambda)(t-2-\lambda-\ell)}(-1)^{q-t+\lambda+1} d^{q-2 t+\lambda+\ell+2}\left(\begin{array}{c}
q-t+\lambda+1 \\
q-2 t+\lambda+\ell+2
\end{array}\right) \\
+(-1)^{q-t+j} d^{q-2 t+\lambda+\ell+1}\left(\begin{array}{c}
q-t+\lambda \\
q-2 t+\lambda+\ell+1
\end{array}\right)\left(\begin{array}{c}
n+1 \\
t-1-\lambda
\end{array}\right)
\end{array}
$$

for $j=0,1, \cdots, t-1$. Put (2.66) back into (2.65) and use (2.67) to reiterate the procedure as (2.65). That is,

$$
\begin{aligned}
& \text { (2.68) } \sum_{\ell=0}^{t-1} \sum_{j=t-\ell}^{q-\ell} \alpha_{(q-j)(q-j-\ell)}(-1)^{j-1} d^{j+\ell-t}\left(\begin{array}{c}
j-1 \\
j+\ell-t
\end{array}\right) \\
&= \sum_{j=0}^{t-1} \sum_{\ell=0}^{t-1-j} \alpha_{(t-1-j)(t-1-j-\ell)}(-1)^{q-t+j} d^{q-2 t+j+\ell+1}\left(\begin{array}{c}
q-t+j \\
q-2 t+j+\ell+1
\end{array}\right) \\
&+(q-t) \sum_{\ell=0}^{t-1} \alpha_{(t-1)(t-1-\ell)}(-1)^{q-t} d^{q-2 t+\ell+1}\left(\begin{array}{c}
q-t \\
q-2 t+\ell+1
\end{array}\right) \\
&+\sum_{j=t}^{q-t} j(-1)^{j-1} d^{j-t}\left(\begin{array}{c}
n+1 \\
q-j
\end{array}\right)\left(\begin{array}{c}
j-1 \\
j-t
\end{array}\right) \\
&=\sum_{j=\lambda}^{t-1} \sum_{\ell=0}^{t-1-j} \alpha_{(t-1-j)(t-1-j-\ell)}(-1)^{q-t+j} d^{q-2 t+j+\ell+1}\left(\begin{array}{c}
q-t+j \\
q-2 t+j+\ell+1
\end{array}\right) \\
&+(q-t+\lambda) \sum_{\ell=0}^{t-1-\lambda} \alpha_{(t-1-\lambda)(t-1-\lambda-\ell)}(-1)^{q-t+\lambda} d^{q-2 t+\lambda+\ell+1}\left(\begin{array}{c}
q-t+\lambda \\
q-2 t+\lambda+\ell+1
\end{array}\right) \\
&+\sum_{j=t}^{q-t+\lambda} j(-1)^{j-1} d^{j-t}\left(\begin{array}{c}
n+1 \\
q-j
\end{array}\right)\left(\begin{array}{c}
j-1 \\
j-t
\end{array}\right)
\end{aligned}
$$

for $\lambda=1, \cdots, t-1$.

By substituting $\lambda=t-1$ in (2.68), we get

$$
\begin{aligned}
& \sum_{\ell=0}^{t-1} \sum_{j=t-\ell}^{q-\ell} \alpha_{(q-j)(q-j-\ell)}(-1)^{j-1} d^{j+\ell-t}\left(\begin{array}{c}
j-1 \\
j+\ell-t
\end{array}\right) \\
& =(-1)^{q-1} d^{q-t}\left(\begin{array}{c}
q-1 \\
q-t
\end{array}\right)+(q-1)(-1)^{q-1} d^{q-t}\left(\begin{array}{l}
q-1 \\
q-t
\end{array}\right) \\
& +\sum_{j=t}^{q-1} j(-1)^{j-1} d^{j-t}\left(\begin{array}{c}
n+1 \\
q-j
\end{array}\right)\left(\begin{array}{c}
j-1 \\
j-t
\end{array}\right) \\
& =\sum_{j=t}^{q} j(-1)^{j-1} d^{j-t}\left(\begin{array}{c}
n+1 \\
q-j
\end{array}\right)\left(\begin{array}{c}
j-1 \\
j-t
\end{array}\right)=-t \alpha_{q(q-t)} .
\end{aligned}
$$


By substituting (2.69) into (2.56), we get the coefficient for $\operatorname{div}(X)$ :

$$
\begin{gathered}
\sum_{j=1}^{q}(-1)^{j-1} \psi_{j-1} \sum_{k=0}^{q-j} \alpha_{(q-j) k} \omega^{k}\left(\frac{\sqrt{-1}}{2 \pi} \partial \bar{\partial} \xi\right)^{q-j-k} \\
=\sum_{t=1}^{q} \sum_{\ell=0}^{t-1} \sum_{j=t-\ell}^{q-\ell} \alpha_{(q-j)(q-j-\ell)}(-1)^{j-1} d^{j+\ell-t}\left(\begin{array}{c}
j-1 \\
j+\ell-t
\end{array}\right) \omega^{q-t}\left(\frac{\sqrt{-1}}{2 \pi} \partial \bar{\partial} \xi\right)^{t-1} \\
=-\sum_{t=1}^{q} t \alpha_{q(q-t)} \omega^{q-t}\left(\frac{\sqrt{-1}}{2 \pi} \partial \bar{\partial} \xi\right)^{t-1} \\
=-\sum_{t=0}^{q-1}(q-t) \alpha_{q t} \omega^{t}\left(\frac{\sqrt{-1}}{2 \pi} \partial \bar{\partial} \xi\right)^{q-t-1}
\end{gathered}
$$

The coefficient for $\theta$ in (2.47) is

$$
\begin{gathered}
-(n+1) \sum_{j=2}^{q}(-1)^{j-1}\left(\psi_{j-1}-\omega^{j-1}\right) \sum_{k=0}^{q-j} \alpha_{(q-j) k} \omega^{k}\left(\frac{\sqrt{-1}}{2 \pi} \partial \bar{\partial} \xi\right)^{q-j-k} \\
=-(n+1) \sum_{j=2}^{q}(-1)^{j-1} \psi_{j-1} \sum_{k=0}^{q-j} \alpha_{(q-j) k} \omega^{k}\left(\frac{\sqrt{-1}}{2 \pi} \partial \bar{\partial} \xi\right)^{q-j-k} \\
+(n+1) \sum_{j=2}^{q}(-1)^{j-1} \omega^{j-1} \sum_{k=0}^{q-j} \alpha_{(q-j) k} \omega^{k}\left(\frac{\sqrt{-1}}{2 \pi} \partial \bar{\partial} \xi\right)^{q-j-k}
\end{gathered}
$$

Using (2.24) in the second part of (2.71), we get

$$
\begin{aligned}
& (n+1) \sum_{j=1}^{q}(-1)^{j} \sum_{k=0}^{q-j} \alpha_{(q-j) k} \omega^{k+j-1}\left(\frac{\sqrt{-1}}{2 \pi} \partial \bar{\partial} \xi\right)^{q-j-k} \\
& =-(n+1) \sum_{t=0}^{q-1} \sum_{k=0}^{t} \alpha_{(q-t-1+k) k}(-1)^{t-k} \omega^{t}\left(\frac{\sqrt{-1}}{2 \pi} \partial \bar{\partial} \xi\right)^{q-t-1} \\
& =(-1)^{q}(n+1) \sum_{t=0}^{q-1} \sum_{k=0}^{t}\left(\begin{array}{c}
q-1-k \\
t-k
\end{array}\right)\left(\begin{array}{l}
n \\
k
\end{array}\right)(-1)^{k} d^{t-k} \omega^{t}\left(\frac{\sqrt{-1}}{2 \pi} \partial \bar{\partial} \xi\right)^{q-t-1} .
\end{aligned}
$$


Use (2.24) to compute

$$
\begin{gathered}
d(q-t) \alpha_{q t}-(t+1) \alpha_{q(t+1)} \\
=(-1)^{q} d(q-t) \sum_{k=0}^{t}\left(\begin{array}{c}
q-k \\
t-k
\end{array}\right)\left(\begin{array}{c}
n+1 \\
k
\end{array}\right) d^{t-k}(-1)^{k} \\
-(-1)^{q}(t+1) \sum_{k=0}^{t+1}\left(\begin{array}{c}
q-k \\
t+1-k
\end{array}\right)\left(\begin{array}{c}
n+1 \\
k
\end{array}\right) d^{t+1-k}(-1)^{k} \\
=(-1)^{q} \sum_{k=0}^{t-1}(-1)^{k+1}\left((q-t)\left(\begin{array}{c}
q-1-k \\
t-1-k
\end{array}\right)-(t+1)\left(\begin{array}{c}
q-1-k \\
t-k
\end{array}\right)\right)\left(\begin{array}{c}
n+1 \\
k+1
\end{array}\right) \\
+(-1)^{q+t}(t+1)\left(\begin{array}{c}
q-1-t \\
0
\end{array}\right)\left(\begin{array}{c}
n+1 \\
t+1
\end{array}\right) \\
=(-1)^{q} \sum_{k=0}^{t}(-1)^{k}(n+1)\left(\begin{array}{c}
q-1-k \\
t-k
\end{array}\right)\left(\begin{array}{l}
n \\
k
\end{array}\right) .
\end{gathered}
$$

Comparing the coefficients in (2.73) and (2.72), we get

$$
\begin{aligned}
(n+1) \sum_{j=1}^{q}(-1)^{j} & \sum_{k=0}^{q-j} \alpha_{(q-j) k} \omega^{k+j-1}\left(\frac{\sqrt{-1}}{2 \pi} \partial \bar{\partial} \xi\right)^{q-j-k} \\
& =-\sum_{t=0}^{q-1}\left(d(q-t) \alpha_{q t}-(t+1) \alpha_{q(t+1)}\right) \omega^{t}\left(\frac{\sqrt{-1}}{2 \pi} \partial \bar{\partial} \xi\right)^{q-t-1}
\end{aligned}
$$

Substituting (2.70) and (2.74) in (2.72), the coefficient for $\theta$ in (2.47) is

$$
\sum_{t=0}^{q-1}\left((n+1-d)(q-t) \alpha_{q t}+(t+1) \alpha_{q(t+1)}\right) \omega^{t}\left(\frac{\sqrt{-1}}{2 \pi} \partial \bar{\partial} \xi\right)^{q-t-1} .
$$

By using Sublemma 2.4 and (2.49), the coefficient for $-\partial \bar{\partial} \theta$ in (2.47) is

$$
\begin{aligned}
& \frac{\sqrt{-1}}{2 \pi} \sum_{j=2}^{q}(-1)^{j-1}\left(n \sum_{\ell=0}^{j-2}\left(d \omega+\frac{\sqrt{-1}}{2 \pi} \partial \bar{\partial} \xi\right)^{j-2-\ell} \omega^{\ell}+\omega^{j-2}\right) \\
& \cdot \sum_{k=0}^{q-j} \alpha_{(q-j) k} \omega^{k}\left(\frac{\sqrt{-1}}{2 \pi} \partial \bar{\partial} \xi\right)^{q-j-k}=\sum_{j=2}^{q}(-1)^{j-1}\left(n \zeta_{j-2}+\omega^{j-2}\right) \wedge \eta_{q-j}
\end{aligned}
$$

where

$$
\begin{aligned}
\eta_{q-j} & =\sum_{k=0}^{q-j} \alpha_{(q-j) k} \omega^{k}\left(\frac{\sqrt{-1}}{2 \pi} \partial \bar{\partial} \xi\right)^{q-j-k}, \\
\zeta_{j-2} & =\frac{\sqrt{-1}}{2 \pi} \sum_{k=0}^{j-2}\left(d \omega+\frac{\sqrt{-1}}{2 \pi} \partial \bar{\partial} \xi\right)^{j-2-k} \omega^{k} .
\end{aligned}
$$


By using Sublemma 2.4 and (2.49), the coefficient for $\partial \bar{\partial} \triangle \theta$ in (2.47) is

$$
\begin{array}{r}
\frac{\sqrt{-1}}{2 \pi} \sum_{j=2}^{q}(-1)^{j-1} \sum_{\ell=0}^{j-2}\left(d \omega+\frac{\sqrt{-1}}{2 \pi} \partial \bar{\partial} \xi\right)^{j-2-\ell} \omega^{\ell} \sum_{k=0}^{q-j} \alpha_{(q-j) k} \omega^{k}\left(\frac{\sqrt{-1}}{2 \pi} \partial \bar{\partial} \xi\right)^{q-j-k} \\
=\sum_{j=2}^{q}(-1)^{j-1} \zeta_{j-2} \wedge \eta_{q-j}
\end{array}
$$

By adding $-\operatorname{div}(X) \cdot(2.70)+\theta \cdot(2.75)+\partial \bar{\partial} \theta \wedge(2.76)+\partial \bar{\partial} \triangle \theta \wedge(2.77)$, we obtain $q \tilde{P}^{q}(\nabla X, \Theta, \cdots, \Theta)$.

Lemma 2.5. The Hodge decomposition of equation (2.44) can be computed as follows:

$$
-q \tilde{P}^{q}(\nabla X, \Theta, \cdots, \Theta)+q \alpha_{q q} \theta \omega^{q-1}-i(X) \partial f_{q}=-\kappa \alpha_{q(q-1)} \omega^{q-1}+\bar{\partial} \varphi_{q},
$$

where

$$
\begin{aligned}
\varphi_{q}= & \kappa \frac{\sqrt{-1}}{2 \pi} \sum_{k=0}^{q-2}(q-k) \omega^{k} \wedge(\partial \xi) \wedge\left(\frac{\sqrt{-1}}{2 \pi} \partial \bar{\partial} \xi\right)^{q-k-2} \\
& +\frac{\sqrt{-1}}{2 \pi} \sum_{k=1}^{q-1} k \alpha_{q k} \theta(\partial \xi) \wedge \omega^{k-1} \wedge\left(\frac{\sqrt{-1}}{2 \pi} \partial \bar{\partial} \xi\right)^{q-k-1} \\
& -\frac{\sqrt{-1}}{2 \pi} \sum_{k=0}^{q-1} \alpha_{q k}(q-k-1) X(\xi) \partial \xi \omega^{k} \wedge\left(\frac{\sqrt{-1}}{2 \pi} \partial \bar{\partial} \xi\right)^{q-k-2} \\
& -\sum_{j=1}^{q}(-1)^{j-1}\left[(\partial \theta) \wedge\left(\omega^{j-1}+n \zeta_{j-2}\right)-(\partial \triangle \theta) \wedge \zeta_{j-2}\right] \wedge \eta_{q-j}
\end{aligned}
$$

is a globally defined form.

Proof. In (2.38), the potential form is obtained:

$$
f_{q}=\sum_{k=0}^{q-1} \alpha_{q k} \xi \omega^{k} \wedge\left(\frac{\sqrt{-1}}{2 \pi} \partial \bar{\partial} \xi\right)^{q-k-1}
$$

for $q=1, \cdots, n-1$. By direction computation, we get

$$
\begin{aligned}
i(X) \partial f_{q}=\sum_{k=0}^{q-1} \alpha_{q k} X(\xi) \omega^{k} \wedge\left(\frac{\sqrt{-1}}{2 \pi} \partial \bar{\partial} \xi\right)^{q-k-1} \\
+\sum_{k=1}^{q-1} k \alpha_{q k} \frac{\sqrt{-1}}{2 \pi}(\partial \xi) \wedge(\bar{\partial} \theta) \wedge \omega^{k-1}\left(\frac{\sqrt{-1}}{2 \pi} \partial \bar{\partial} \xi\right)^{q-k-1} \\
\quad-\sum_{k=0}^{q-2}(q-k-1) \alpha_{q k} \frac{\sqrt{-1}}{2 \pi}(\partial \xi) \wedge(\bar{\partial} X(\xi)) \wedge \omega^{k} \wedge\left(\frac{\sqrt{-1}}{2 \pi} \partial \bar{\partial} \xi\right)^{q-k-2} .
\end{aligned}
$$


Using Lemma 2.4 and (2.80), we obtain

$$
\begin{aligned}
-q \tilde{P}^{q} & (\nabla X, \Theta, \cdots, \Theta)+q \alpha_{q q} \theta \omega^{q-1}-i(X) \partial f_{q} \\
& =\operatorname{div}(X) \sum_{k=0}^{q-1}(q-k) \alpha_{q k} \omega^{k} \wedge\left(\frac{\sqrt{-1}}{2 \pi} \partial \bar{\partial} \xi\right)^{q-1-k} \\
& -\theta \sum_{k=0}^{q-1}\left[(q-k)(n+1-d) \alpha_{q k}+(k+1) \alpha_{q(k+1)}\right] \omega^{k} \wedge\left(\frac{\sqrt{-1}}{2 \pi} \partial \bar{\partial} \xi\right)^{q-1-k} \\
& +\sum_{j=1}^{q}(-1)^{j-1}\left[(\partial \bar{\partial} \theta) \wedge\left(\omega^{j-1}+n \zeta_{j-2}\right)-(\partial \bar{\partial} \triangle \theta) \wedge \zeta_{j-2}\right] \wedge \eta_{q-j} \\
& +q \alpha_{q q} \theta \omega^{q-1}-X(\xi) \sum_{k=0}^{q-1}[(q-k)-(q-k-1)] \alpha_{q k} \omega^{k} \wedge\left(\frac{\sqrt{-1}}{2 \pi} \partial \bar{\partial} \xi\right)^{q-1-k} \\
& -\frac{\sqrt{-1}}{2 \pi} \sum_{k=1}^{q-1} k \alpha_{q k}(\partial \xi) \wedge(\bar{\partial} \theta) \wedge \omega^{k-1}\left(\frac{\sqrt{-1}}{2 \pi} \partial \bar{\partial} \xi\right)^{q-k-1} \\
& +\frac{\sqrt{-1}}{2 \pi} \sum_{k=0}^{q-2}(q-k-1) \alpha_{q k}(\partial \xi) \wedge(\bar{\partial} X(\xi)) \wedge \omega^{k} \wedge\left(\frac{\sqrt{-1}}{2 \pi} \partial \bar{\partial} \xi\right)^{q-k-2} .
\end{aligned}
$$

In fact, we have the formula [12, Theorem 4.1]

$$
\operatorname{div}(X)-X(\xi)-(n-d+1) \theta=-\kappa .
$$

By using (2.82) in (2.81), we have

$$
\begin{aligned}
-q \tilde{P}^{q} & (\nabla X, \Theta, \cdots, \Theta)+q \alpha_{q q} \theta \omega^{q-1}-i(X) \partial f_{q} \\
= & -\kappa \sum_{k=0}^{q-1}(q-k) \alpha_{q k} \omega^{k} \wedge\left(\frac{\sqrt{-1}}{2 \pi} \partial \bar{\partial} \xi\right)^{q-1-k} \\
& -\theta \sum_{k=1}^{q-1} k \alpha_{q k} \omega^{k-1} \wedge\left(\frac{\sqrt{-1}}{2 \pi} \partial \bar{\partial} \xi\right)^{q-1-k} \\
& -\frac{\sqrt{-1}}{2 \pi} \sum_{k=1}^{q-1} k \alpha_{q k}(\partial \xi) \wedge(\bar{\partial} \theta) \wedge \omega^{k-1}\left(\frac{\sqrt{-1}}{2 \pi} \partial \bar{\partial} \xi\right)^{q-k-1} \\
+ & \sum_{j=1}^{q}(-1)^{j-1}\left[(\partial \bar{\partial} \theta) \wedge\left(\omega^{j-1}+n \zeta_{j-2}\right)-(\partial \bar{\partial} \triangle \theta) \wedge \zeta_{j-2}\right] \wedge \eta_{q-j} \\
+ & X(\xi) \sum_{k=0}^{q-2}(q-k-1) \alpha_{q k} \omega^{k} \wedge\left(\frac{\sqrt{-1}}{2 \pi} \partial \bar{\partial} \xi\right)^{q-1-k} \\
+ & \frac{\sqrt{-1}}{2 \pi} \sum_{k=0}^{q-2}(q-k-1) \alpha_{q k}(\partial \xi) \wedge(\bar{\partial} X(\xi)) \wedge \omega^{k} \wedge\left(\frac{\sqrt{-1}}{2 \pi} \partial \bar{\partial} \xi\right)^{q-k-2} .
\end{aligned}
$$

We can decompose (2.83) as $-\kappa \alpha_{q(q-1)} \omega^{q-1}+\bar{\partial} \varphi_{q}$, where $\varphi_{q}$ is given in (2.79). Since $\omega^{q}$ is harmonic, the harmonic part of (2.83) is $-\kappa \alpha_{q(q-1)} \omega^{q}$. 
Lemma 2.6. We define $q \tilde{P}^{q}(\nabla X, \Theta, \cdots, \Theta)$ in (2.47). For $q=1, \cdots, n-1$, we have

$$
\int_{M} q \tilde{P}^{q}(\nabla X, \Theta, \cdots, \Theta) \wedge \omega_{M}^{n-q}=0
$$

where $\omega_{M}=(n+1-d) \omega$.

Proof. Consider

$$
\begin{aligned}
0= & \int_{M} L_{X}\left(P^{q-1}(\Theta) \wedge \omega^{n-q}\right) \\
= & \int_{M}\left(L_{X} P^{q-1}(\Theta)\right) \wedge \omega^{n-q}+\int_{M} P^{q-1}(\Theta) \wedge L_{X}\left(\omega^{n-q}\right) \\
= & -(q-1) \frac{\sqrt{-1}}{2 \pi} \int_{M} \partial \bar{\partial} \tilde{P}^{q-1}(\nabla X, \Theta, \cdots, \Theta) \wedge \omega^{n-q} \\
& -(n-q) \frac{\sqrt{-1}}{2 \pi} \int_{M} P^{q-1}(\Theta) \wedge \partial \bar{\partial} \theta \wedge \omega^{n-q-1} .
\end{aligned}
$$

By Stoke's Theorem, we have $\int_{M} \partial \bar{\partial} \tilde{P}^{q-1}(\nabla X, \Theta, \cdots, \Theta) \wedge \omega^{n-q}=0$. By using (2.13) and (2.42), we compute that

$$
\begin{aligned}
& -(n-q) \frac{\sqrt{-1}}{2 \pi} \partial \bar{\partial} \theta \wedge P^{q-1}(\Theta) \wedge \omega^{n-q-1} \\
& =-\frac{(n-q)(n-1-q) !}{(q-1) !(n-1) !} \sum_{\sigma \in S_{q-1}, \tau \in S_{q}} \sum_{i_{1}, j_{1}, k_{1} \cdots, k_{q-1}, i_{q}, j_{q}=2}^{n} \operatorname{sgn}(\sigma) \operatorname{sgn}(\tau) \\
& =\frac{(n-q) !}{(q-1) !(n-1) !} \sum_{\sigma \in S_{q-1}, \tau \in S_{q} i_{1}, k_{1}, s_{1}, \cdots, s_{q-1}, i_{q}, k_{q-1}=2} \sum_{j_{j_{1}} \theta R_{k_{1} i_{2} \bar{j}_{2}}^{k_{\sigma(1)}} \cdots R_{k_{q-1} i_{q} \bar{j}_{q}}^{k_{\sigma(q-1)}} \tilde{g}^{i_{\tau(1)} \bar{j}_{1}} \cdots \tilde{g}^{i_{\tau(q)} \bar{j}_{q}} \omega^{n-1}}^{n} \operatorname{sgn}(\sigma) \operatorname{sgn}(\tau) \\
& \cdot X_{i_{1}}^{i_{\tau(1)}} R_{i_{2} k_{1} \bar{s}_{1}}^{i_{\tau(2)}} \cdots R_{i_{q} k_{q-1} \bar{s}_{q-1}}^{i_{\tau(q)}} \tilde{g}^{k_{\sigma(1)} \bar{s}_{1}} \cdots \tilde{g}^{k_{\sigma(q-1)} \bar{s}_{q-1}} \omega^{n-1} .
\end{aligned}
$$

By definition, we have

$$
\begin{aligned}
\tilde{P}^{q}(\nabla X, \Theta, \cdots, \Theta)=\frac{1}{q !}\left(\frac{\sqrt{-1}}{2 \pi}\right)^{q-1} \sum_{\tau \in S_{q} i_{1}, k_{1}, s_{1} \cdots, k_{q-1}, s_{q-1}, i_{q}=2} \operatorname{sgn}(\tau) \\
\cdot X_{i_{1}}^{i_{\tau(1)}} R_{i_{2} k_{1} \bar{s}_{1}}^{i_{\tau(2)}} \cdots R_{i_{q} k_{q-1} \bar{s}_{q-1}}^{i_{\tau(q)}} d z_{k_{1}} \wedge d \bar{z}_{s_{1}} \wedge \cdots d z_{k_{q-1}} \wedge d \bar{z}_{s_{q-1}} .
\end{aligned}
$$

By comparing (2.86) and (2.87), we obtain that

$$
-(n-q) \frac{\sqrt{-1}}{2 \pi} \partial \bar{\partial} \theta \wedge P^{q-1}(\Theta) \wedge \omega^{n-q-1}=q \tilde{P}^{q}(\nabla X, \Theta, \cdots, \Theta) \wedge \omega^{n-q} .
$$

Therefore, we have

$$
\int_{M} q \tilde{P}^{q}(\nabla X, \Theta, \cdots, \Theta) \wedge \omega_{M}^{n-q}=\int_{M} L_{X}\left(P^{q-1}(\Theta) \wedge \omega_{M}^{n-q}\right)=0 .
$$

Lemma 2.6 can be considered as a result of a generalized divergence theorem. Please see the Appendix for a further study. 
Proof of Theorem 1.1. From (2.45), we can compute the $q$-th Bando-Futaki invariant by

$$
\begin{aligned}
\int_{M} L_{X} f_{q} \wedge \omega_{M}^{n-q}= & (n+1-d)^{n-q}\left(-C(q) \int_{M} \omega^{n-1}+q \alpha_{q q} \int_{M} \theta \omega^{n-1}\right) \\
& -q \int_{M} \tilde{P}^{q}(\nabla X, \Theta, \cdots, \Theta) \wedge \omega_{M}^{n-q}-\int_{M} \bar{\partial} \varphi_{q} \wedge \omega_{M}^{n-q} .
\end{aligned}
$$

From Lemma 2.5 we have $C(q)=-\kappa \alpha_{q(q-1)}$. By [12, Theorem 5.1], we have $\int_{M} \theta \omega^{n-1}=\frac{\kappa}{n}$. From Lemma 2.6, we have $q \int_{M} \tilde{P}^{q}(\nabla X, \Theta, \cdots, \Theta) \wedge \omega_{M}^{n-q}=0$. In addition, the divergence theorem implies that $\int_{M} \bar{\partial} \varphi_{q} \wedge \omega_{M}^{n-q}=0$. With this information, we get

$$
\int_{M} L_{X} f_{q} \wedge \omega_{M}^{n-q}=\kappa(n+1-d)^{n-q}\left(\alpha_{q q} \frac{q}{n}+d \alpha_{q(q-1)}\right) .
$$

Using (2.24), the $q$-th Bando-Futaki invariant can be computed directly by

$$
\begin{aligned}
\mathcal{F}_{q}(X) & =-(n+1-d)^{n-q} \frac{(d-1)}{n} \sum_{j=0}^{q-1}(-d)^{j}(j+1)(q-j)\left(\begin{array}{c}
n+1 \\
q-j
\end{array}\right) \kappa \\
& =-(n+1-d)^{n-q} \frac{(d-1)(n+1)}{n} \sum_{j=0}^{q-1}(-d)^{j}(j+1)\left(\begin{array}{c}
n \\
q-1-j
\end{array}\right) \kappa .
\end{aligned}
$$

Corollary 2.1. With the notation as in Theorem 1.1, if $d=1$, all the Bando-Futaki invariants are zero.

Corollary 2.2. With the notation as in Theorem 1.1, if $M$ is a cubic surface in $\mathbb{C P}^{3}$, then the Bando-Futaki invariants are

$$
\mathcal{F}_{1}(X)=-\frac{8}{3} \kappa \quad \text { and } \quad \mathcal{F}_{2}(X)=16 \kappa .
$$

The following example is due to [4] and [12].

Example 2.1. Let

$$
M=\left\{Z \in \mathbb{C P}^{3} \mid F(Z)=Z_{0} Z_{1}^{2}+Z_{2}^{2} Z_{3}-Z_{2} Z_{3}^{2}=0\right\}
$$

be a hypersurface in $\mathbb{C P}^{3}$, where $Z=\left[Z_{0}, Z_{1}, Z_{2}, Z_{3}\right]$ are the homogeneous coordinates of $\mathbb{C P}^{3} . M$ is a Kähler orbifold with $c_{1}(M)>0$. Let

$$
X=-7 Z_{0} \frac{\partial}{\partial Z_{0}}+5 Z_{1} \frac{\partial}{\partial Z_{1}}+Z_{2} \frac{\partial}{\partial Z_{2}}+Z_{3} \frac{\partial}{\partial Z_{3}}
$$

be a holomorphic vector field in $\mathbb{C P}^{3}$, which satisfies $X F=3 F$. The Bando-Futaki invariants are

$$
\mathcal{F}_{1}(X)=-8 \quad \text { and } \quad \mathcal{F}_{2}(X)=48
$$

Example 2.2. Let

$$
M=\left\{Z \in \mathbb{C P}^{4} \mid F(Z)=Z_{0}^{2}+Z_{1}^{2}+Z_{2}^{2}+Z_{3}^{2}+Z_{4}^{2}=0\right\}
$$

be a hypersurface in $\mathbb{C P}^{4}$. Let

$$
X=Z_{0} \frac{\partial}{\partial Z_{0}}+Z_{1} \frac{\partial}{\partial Z_{1}}+Z_{2} \frac{\partial}{\partial Z_{2}}+Z_{3} \frac{\partial}{\partial Z_{3}}+Z_{4} \frac{\partial}{\partial Z_{4}}
$$


be a holomorphic vector field on $\mathbb{C P}^{4}$, which satisfies

$$
X(F)=2 F \text {. }
$$

The Bando-Futaki invariants are

$$
\mathcal{F}_{1}(X)=-270, \quad \mathcal{F}_{2}(X)=135, \quad \text { and } \quad F_{3}(X)=-\frac{285}{2} .
$$

\section{Chen And Tian's holomorphic invariants}

The following holomorphic invariants were introduced by Chen and Tian [3]. We prove that they are the Futaki invariants.

Definition 3.1. Let $M$ be an $n$-dimensional simply connected Kähler manifold with positive first Chern class $c_{1}(M)$. Let $\omega$ be a Kähler form such that $[\omega] \in c_{1}(M)$. There exists a smooth function $\theta_{X}$ such that $i(X) \omega=\frac{\sqrt{-1}}{2 \pi} \bar{\partial} \theta_{X}{ }^{3}$ Define

$$
\begin{aligned}
\mathcal{F}_{k}(X, \omega)= & (n-k) \int_{M} \theta_{X} \omega^{n}+(k+1) \int_{M} \Delta \theta_{X} \operatorname{Ric}(\omega)^{k} \wedge \omega^{n-k} \\
& -(n-k) \int_{M} \theta_{X} \operatorname{Ric}(\omega)^{k+1} \wedge \omega^{n-k-1} .
\end{aligned}
$$

These new holomorphic invariants are independent of the choices of the Kähler metrics in the Kähler class $[\omega]$, which were shown in [3].

Proof of Theorem 1.2. There exists a smooth real-valued function $f$ over $M$, such that

$$
\operatorname{Ric}(\omega)-\omega=\frac{\sqrt{-1}}{2 \pi} \partial \bar{\partial} f .
$$

Taking the inner derivative on both sides, we have

$$
\operatorname{div} X+\theta_{X}+X(f)=\beta
$$

where $\beta$ is a constant if $M$ is compact. We need the following two formulas:

$$
\begin{aligned}
0 & =\int_{M}\left(i(X)\left[\partial f(\partial \bar{\partial} f)^{j-1} \omega^{n-j+1}\right]\right) \\
& =j \int_{M} X(f)(\partial \bar{\partial} f)^{j-1} \omega^{n-j+1}+(n-j+1) \int_{M} \bar{\partial} \theta_{X} \partial f(\partial \bar{\partial} f)^{j-1} \omega^{n-j} \\
& =j \int_{M} X(f)(\partial \bar{\partial} f)^{j-1} \omega^{n-j+1}+(n-j+1) \int_{M} \theta_{X}(\partial \bar{\partial} f)^{j} \omega^{n-j}
\end{aligned}
$$

for $1 \leq j \leq k+1$, and

$$
\operatorname{div} X=\Delta \theta_{X}+c,
$$

where $c$ is a constant and $\Delta \theta_{X}=g^{i \bar{j}} \partial_{i} \bar{\partial}_{j} \theta_{X}$ if $\omega=\frac{\sqrt{-1}}{2 \pi} \sum_{i, j=1}^{n} g_{i j} d z_{i} \wedge d \bar{z}_{j}$. By using (3.2), the new holomorphic invariants can be written as

$$
\begin{aligned}
\mathcal{F}_{k}(X, \omega)= & (k+1) \int_{M} \Delta \theta_{X} \sum_{i=1}^{k}\left(\begin{array}{c}
k \\
i
\end{array}\right)\left(\frac{\sqrt{-1}}{2 \pi} \partial \bar{\partial} f\right)^{i} \omega^{n-i} \\
& -(n-k) \int_{M} \theta_{X} \sum_{i=1}^{k+1}\left(\begin{array}{c}
k+1 \\
i
\end{array}\right)\left(\frac{\sqrt{-1}}{2 \pi} \partial \bar{\partial} f\right)^{i} \omega^{n-i}
\end{aligned}
$$

\footnotetext{
${ }^{3}$ In order to maintain the definition as in the original paper, we have opposite signs of the notation for $\theta_{X}$ and $\Delta$ from what we used in the previous section.
} 
For each $1 \leq i \leq k+1$, using $(n-i+1)=(n-k)+(k-i-1)$ and (3.4), we get

$$
\begin{aligned}
& -(n-k) \int_{M} \theta_{X}\left(\frac{\sqrt{-1}}{2 \pi} \partial \bar{\partial} f\right)^{i} \omega^{n-i} \\
& \quad=(k-i-1) \int_{M} \theta_{X}\left(\frac{\sqrt{-1}}{2 \pi} \partial \bar{\partial} f\right)^{i} \omega^{n-i}+i \int_{M} X(f)(\partial \bar{\partial} f)^{i-1} \omega^{n-i+1} .
\end{aligned}
$$

By using (3.5) and (3.6), we have

$$
\begin{aligned}
\mathcal{F}_{k}(X, \omega)= & (k+1) \int_{M} \operatorname{div} X \sum_{i=1}^{k}\left(\begin{array}{c}
k \\
i
\end{array}\right)\left(\frac{\sqrt{-1}}{2 \pi} \partial \bar{\partial} f\right)^{i} \omega^{n-i} \\
& +\int_{M} \sum_{i=1}^{k+1} i\left(\begin{array}{c}
k+1 \\
i
\end{array}\right) X(f)\left(\frac{\sqrt{-1}}{2 \pi} \partial \bar{\partial} f\right)^{i-1} \omega^{n-i+1} \\
& +\int_{M} \sum_{i=1}^{k+1}(k-i+1)\left(\begin{array}{c}
k+1 \\
i
\end{array}\right) \theta_{X}\left(\frac{\sqrt{-1}}{2 \pi} \partial \bar{\partial} f\right)^{i} \omega^{n-i}
\end{aligned}
$$

Since $\sum_{i=1}^{k+1} i\left(\begin{array}{c}k+1 \\ i\end{array}\right)=\sum_{i=0}^{k}(i+1)\left(\begin{array}{c}k+1 \\ i+1\end{array}\right)=(k+1)+\sum_{i=1}^{k}(k+1)\left(\begin{array}{c}k \\ i\end{array}\right)$, we divide the term with $X(f)$ in (3.7) into two parts and combine one part with $\operatorname{div}(X)$. That is,

$$
\begin{aligned}
\mathcal{F}_{k}(X, \omega)= & (k+1) \int_{M}(\operatorname{div} X+X(f)) \sum_{i=1}^{k}\left(\begin{array}{c}
k \\
i
\end{array}\right)\left(\frac{\sqrt{-1}}{2 \pi} \partial \bar{\partial} f\right)^{i} \omega^{n-i} \\
& +(k+1) \int_{M} X(f) \omega^{n} \\
& +\int_{M} \sum_{i=1}^{k+1}(k+1)\left(\begin{array}{c}
k \\
i
\end{array}\right) \theta_{X}\left(\frac{\sqrt{-1}}{2 \pi} \partial \bar{\partial} f\right)^{i} \omega^{n-i} .
\end{aligned}
$$

By using (3.3) in (3.8), we get

$$
\begin{aligned}
\mathcal{F}_{k}(X, \omega)= & (k+1) \int_{M} X(f) \omega^{n}-(k+1) \int_{M} \theta_{X} \sum_{i=1}^{k}\left(\begin{array}{c}
k \\
i
\end{array}\right)\left(\frac{\sqrt{-1}}{2 \pi} \partial \bar{\partial} f\right)^{i} \omega^{n-i} \\
& +\int_{M} \sum_{i=1}^{k}(k+1)\left(\begin{array}{c}
k \\
i
\end{array}\right) \theta_{X}\left(\frac{\sqrt{-1}}{2 \pi} \partial \bar{\partial} f\right)^{i} \omega^{n-i} \\
= & (k+1) \int_{M} X(f) \omega^{n} .
\end{aligned}
$$

These holomorphic invariants are simply the Futaki invariants on compact Kähler manifolds with $c_{1}(M)>0$. Indeed, Kobayashi [10] proved that a compact Kähler manifold with positive Ricci curvature is simply connected.

\section{Appendix}

Lemma 4.1. For $q=2, \cdots, n-1$, let

$$
Y=\sum_{i_{1}=2}^{n} Y^{i_{1}} \frac{\partial}{\partial z_{i_{1}}} \in T^{1,0}(M) \otimes \wedge^{q-1}\left(T^{1,0}(M)^{*} \otimes{\overline{T^{1,0}(M)}}^{*}\right)
$$


be a holomorphic vector field with $(q-1, q-1)$-valued form, where

$$
Y^{i_{1}}=X^{i_{1}} P^{q-1}(\Theta)+\sum_{j=2}^{q} \sum_{i_{2}, \cdots, i_{j}=2}^{n}(-1)^{j-1} X^{i_{2}} \Theta_{i_{2}}^{i_{3}} \cdots \Theta_{i_{j}}^{i_{1}} P^{q-j}(\Theta) .
$$

Then we have $q \tilde{P}^{q}(\nabla X, \Theta, \cdots, \Theta)=\operatorname{div}(Y)$. For $q=1$, we have $Y=X$ and $\tilde{P}^{1}(\nabla X)=\operatorname{div}(X)$.

Proof. We have

$$
\begin{aligned}
\operatorname{div}(Y)= & \sum_{i_{1}=2}^{n} \nabla_{i_{1}} Y^{i_{1}} \\
= & \sum_{i_{1}=2}^{n} X_{i_{1}}^{i_{1}} P^{q-1}+\sum_{i_{1}=2}^{n} X^{i_{1}} \nabla_{i_{1}} P^{q-1} \\
& +\sum_{j=2}^{q} \sum_{i_{1}, \cdots, i_{j}=2}^{n}(-1)^{j-1} X_{i_{1}}^{i_{2}} \Theta_{i_{2}}^{i_{3}} \cdots \Theta_{i_{j}}^{i_{1}} P^{q-j}(\Theta) \\
& +\sum_{j=2}^{q} \sum_{i_{1}, \cdots, i_{j}=2}^{n}(-1)^{j-1} X^{i_{2}} \nabla_{i_{1}}\left(\Theta_{i_{2}}^{i_{3}} \cdots \Theta_{i_{j}}^{i_{1}}\right) P^{q-j}(\Theta) \\
& +\sum_{j=2}^{q} \sum_{i_{1}, \cdots, i_{j}=2}^{n}(-1)^{j-1} X^{i_{2}} \Theta_{i_{2}}^{i_{3}} \cdots \Theta_{i_{j}}^{i_{1}} \nabla_{i_{1}}\left(P^{q-j}(\Theta)\right) .
\end{aligned}
$$

By using the definition of $q \tilde{P}^{q}(\nabla X, \Theta, \cdots, \Theta)$ in (2.47), we get

$$
\begin{aligned}
\operatorname{div}(Y)= & q \tilde{P}^{q}(\nabla X, \Theta, \cdots, \Theta)+\sum_{i_{1}=2}^{n} X^{i_{1}} \nabla_{i_{1}} P^{q-1} \\
& -\sum_{j=2}^{q} \sum_{i_{1}, \cdots, i_{j}=2}^{n}(-1)^{j} X^{i_{2}} \nabla_{i_{1}}\left(\Theta_{i_{2}}^{i_{3}} \cdots \Theta_{i_{j}}^{i_{1}}\right) P^{q-j}(\Theta) \\
& -\sum_{j=2}^{q} \sum_{i_{1}, \cdots, i_{j}=2}^{n}(-1)^{j} X^{i_{2}} \Theta_{i_{2}}^{i_{3}} \cdots \Theta_{i_{j}}^{i_{1}} \nabla_{i_{1}} P^{q-j}(\Theta) .
\end{aligned}
$$

This is equivalent to showing the following sublemma.

Sublemma 4.1. For $q=2, \cdots, n-1$, we have

$$
\begin{aligned}
\sum_{i_{1}=2}^{n} X^{i_{1}} \nabla_{i_{1}} P^{q-1}= & \sum_{j=2}^{q} \sum_{i_{1}, \cdots, i_{j}=2}^{n}(-1)^{j} X^{i_{2}} \nabla_{i_{1}}\left(\Theta_{i_{2}}^{i_{3}} \cdots \Theta_{i_{j}}^{i_{1}}\right) P^{q-j}(\Theta) \\
& +\sum_{j=2}^{q} \sum_{i_{1}, \cdots, i_{j}=2}^{n}(-1)^{j} X^{i_{2}} \Theta_{i_{2}}^{i_{3}} \cdots \Theta_{i_{j}}^{i_{1}} \nabla_{i_{1}} P^{q-j}(\Theta) .
\end{aligned}
$$

For $q=1$, (4.3) is zero. 
We need two formulas before proving Sublemma 4.1. In fact, equation (4.4) gives

$$
\begin{aligned}
\nabla \Theta & =\sum_{i, j, k=2}^{n} \nabla_{i} \Theta_{j}^{k} \frac{\partial}{\partial z_{k}} \otimes d z_{i} \wedge d z_{j} \\
& =-\sum_{i, j, k=2}^{n} \nabla_{i} \Theta_{j}^{k} \frac{\partial}{\partial z_{k}} \otimes d z_{j} \wedge d z_{i} \\
& =-\sum_{i, j, k=2}^{n} \nabla_{j} \Theta_{i}^{k} \frac{\partial}{\partial z_{k}} \otimes d z_{j} \wedge d z_{i}=-\nabla \Theta .
\end{aligned}
$$

This is the Bianchi identity $\nabla \Theta=0$.

Sublemma 4.2. For $2 \leq i \leq n$ and $q=1, \cdots, n-1$, we compute the covariant derivative on the curvature form and the Chern forms

$$
\begin{aligned}
\nabla_{i} \Theta_{j}^{k} & =\nabla_{j} \Theta_{i}^{k}, \\
\nabla_{i} P^{q}(\Theta) & =\sum_{\ell=1}^{q} \sum_{i_{1}, \cdots, i_{\ell}=2}^{n}(-1)^{\ell-1} \frac{1}{\ell} \nabla_{i}\left(\Theta_{i_{1}}^{i_{2}} \Theta_{i_{2}}^{i_{3}} \cdots \Theta_{i_{\ell}}^{i_{1}}\right) P^{q-\ell}(\Theta),
\end{aligned}
$$

where $\Theta_{j}^{k}=\frac{\sqrt{-1}}{2 \pi} \sum_{s, t=2}^{n} R_{j s \bar{t}}^{k} d z_{s} \wedge d \bar{z}_{t}=-\frac{\sqrt{-1}}{2 \pi} \sum_{s, t=2}^{n} \bar{\partial}_{t} \Gamma_{s j}^{k} d z_{s} \wedge d \bar{z}_{t}$. Since $M$ is Kähler, $\Gamma_{s j}^{k}=\Gamma_{j s}^{k}=\frac{\partial \tilde{g}_{s \bar{\beta}}}{\partial z_{j}} \tilde{g}^{k \bar{\beta}}$.

Proof. First, we compute

$$
\begin{aligned}
\nabla_{i} R_{j s \bar{t}}^{k}= & -\partial_{i} \bar{\partial}_{t} \Gamma_{j s}^{k}+\Gamma_{i \lambda}^{k} R_{j s \bar{t}}^{\lambda}-\Gamma_{i j}^{\lambda} R_{\lambda s \bar{t}}^{k}-\Gamma_{i s}^{\lambda} R_{j \lambda \bar{t}}^{k} \\
= & -\bar{\partial}_{t}\left(\frac{\partial^{2} \tilde{g}_{s \bar{\beta}}}{\partial z_{i} \partial z_{j}} \tilde{g}^{k \bar{\beta}}\right)+\bar{\partial}_{t}\left(\frac{\partial \tilde{g}_{s \bar{\beta}}}{\partial z_{j}} \frac{\partial \tilde{g}_{\alpha \bar{\eta}}}{\partial z_{i}} \tilde{g}^{k \bar{\eta}} \tilde{g}^{\alpha \bar{\beta}}\right) \\
& +\Gamma_{i \lambda}^{k} R_{j s \bar{t}}^{\lambda}-\Gamma_{i j}^{\lambda} R_{\lambda s \bar{t}}^{k}-\Gamma_{i s}^{\lambda} R_{j \lambda \bar{t}}^{k} \\
= & -\bar{\partial}_{t}\left(\frac{\partial^{2} \tilde{g}_{s \bar{\beta}}}{\partial z_{i} \partial z_{j}} \tilde{g}^{k \bar{\beta}}\right)-\Gamma_{i \alpha}^{k} R_{j s \bar{t}}^{\alpha}-\Gamma_{j s}^{\alpha} R_{i \alpha \bar{t}}^{k} \\
& +\Gamma_{i \lambda}^{k} R_{j s \bar{t}}^{\lambda}-\Gamma_{i j}^{\lambda} R_{\lambda s \bar{t}}^{k}-\Gamma_{i s}^{\lambda} R_{j \lambda \bar{t} .}^{k}
\end{aligned}
$$

Similarly, we get

$$
\begin{aligned}
\nabla_{j} R_{i s \bar{t}}^{k}= & -\bar{\partial}_{t}\left(\frac{\partial^{2} \tilde{g}_{s \bar{\beta}}}{\partial z_{j} \partial z_{i}} \tilde{g}^{k \bar{\beta}}\right)-\Gamma_{j \alpha}^{k} R_{i s \bar{t}}^{\alpha}-\Gamma_{i s}^{\alpha} R_{j \alpha \bar{t}}^{k} \\
& +\Gamma_{j \lambda}^{k} R_{i s \bar{t}}^{\lambda}-\Gamma_{j i}^{\lambda} R_{\lambda s \bar{t}}^{k}-\Gamma_{j s}^{\lambda} R_{i \lambda \bar{t}}^{k} .
\end{aligned}
$$

Since $M$ is Kähler, $\Gamma_{i j}^{\lambda}=\Gamma_{j i}^{\lambda}$. Therefore, (4.6) and (4.7) are equal. Then we get (4.4). Next, we prove (4.5) by induction. For $q=1, \nabla_{i} P^{1}(\Theta)=\nabla_{i} \sum_{i_{1}=2}^{n} \Theta_{i_{1}}^{i_{1}}$. Assume that (4.5) is true for $2 \leq k \leq q-1$. By using (2.14), we compute the covariant derivative on the $q$-Chern form:

$$
\begin{aligned}
\nabla_{i} P^{q}(\Theta)= & \frac{1}{q} \sum_{j=1}^{q}(-1)^{j-1} \nabla_{i}\left(\Theta_{i_{1}}^{i_{2}} \Theta_{i_{2}}^{i_{3}} \cdots \Theta_{i_{j}}^{i_{1}}\right) P^{q-j}(\Theta) \\
& +\frac{1}{q} \sum_{j=1}^{q}(-1)^{j-1} \Theta_{i_{1}}^{i_{2}} \Theta_{i_{2}}^{i_{3}} \cdots \Theta_{i_{j}}^{i_{1}} \nabla_{i} P^{q-j}(\Theta) .
\end{aligned}
$$


By using the induction hypothesis on the second term of (4.8), we get

$$
\begin{aligned}
& \frac{1}{q} \sum_{j=1}^{q}(-1)^{j-1} \Theta_{i_{1}}^{i_{2}} \Theta_{i_{2}}^{i_{3}} \cdots \Theta_{i_{j}}^{i_{1}} \nabla_{i} P^{q-j}(\Theta) \\
& \quad=\frac{1}{q} \sum_{j=1}^{q}(-1)^{j-1} \Theta_{i_{1}}^{i_{2}} \cdots \Theta_{i_{j}}^{i_{1}} \sum_{\ell=1}^{q-j}(-1)^{\ell-1} \frac{1}{\ell} \nabla_{i}\left(\Theta_{i_{j+1}}^{i_{j+2}} \cdots \Theta_{i_{j+\ell}}^{i_{j+1}}\right) P^{q-j-\ell}(\Theta) \\
& \quad=\frac{1}{q} \sum_{\ell=1}^{q}(-1)^{\ell-1} \frac{1}{\ell} \nabla_{i}\left(\Theta_{i_{j+1}}^{i_{j+2}} \cdots \Theta_{i_{j+\ell}}^{i_{j+1}}\right) \sum_{j=1}^{q-\ell}(-1)^{j-1} \Theta_{i_{1}}^{i_{2}} \cdots \Theta_{i_{j}}^{i_{1}} P^{q-j-\ell}(\Theta) \\
& \quad=\frac{1}{q} \sum_{\ell=1}^{q}(-1)^{\ell-1} \frac{q-\ell}{\ell} \nabla_{i}\left(\Theta_{i_{1}}^{i_{2}} \cdots \Theta_{i_{\ell}}^{i_{1}}\right) P^{q-\ell}(\Theta) .
\end{aligned}
$$

By substituting (4.9) back into (4.8), we obtain (4.5).

Proof of Sublemma 4.1. The proof is by induction. For $q=2$, by using (4.3), we get

$$
X^{i} \nabla_{i} \Theta_{j}^{j}=X^{i} \nabla_{j} \Theta_{i}^{j}=X^{j} \nabla_{i} \Theta_{j}^{i} .
$$

Assume that (4.3) holds for $k=2, \cdots, q-1$. By using (4.5), we compute

$$
\begin{gathered}
\sum_{i_{1}=2}^{n} X^{i_{1}} \nabla_{i_{1}} P^{q-1}(\Theta)-\sum_{j=2}^{q} \sum_{i_{1}, \cdots, i_{j}=2}^{n}(-1)^{j} X^{i_{2}} \nabla_{i_{1}}\left(\Theta_{i_{2}}^{i_{3}} \cdots \Theta_{i_{j}}^{i_{1}}\right) P^{q-j}(\Theta) \\
\quad-\sum_{j=2}^{q} \sum_{i_{1}, \cdots, i_{j}=2}^{n}(-1)^{j} X^{i_{2}} \Theta_{i_{2}}^{i_{3}} \cdots \Theta_{i_{j}}^{i_{1}} \nabla_{i_{1}} P^{q-j}(\Theta) \\
=\sum_{j=2}^{q} \sum_{i_{1}, \cdots, i_{j}=2}^{n}(-1)^{j} \frac{1}{j-1} X^{i_{1}} \nabla_{i_{1}}\left(\Theta_{i_{2}}^{i_{3}} \cdots \Theta_{i_{j}}^{i_{2}}\right) P^{q-j}(\Theta) \\
\quad-\sum_{j=2}^{q} \sum_{i_{1}, \cdots, i_{j}=2}^{n}(-1)^{j} X^{i_{2}} \nabla_{i_{1}}\left(\Theta_{i_{2}}^{i_{3}} \cdots \Theta_{i_{j}}^{i_{1}}\right) P^{q-j}(\Theta) \\
-\sum_{j=2}^{q} \sum_{i_{1}, \cdots, i_{j}=2}^{n}(-1)^{j} X^{i_{2}} \Theta_{i_{2}}^{i_{3}} \cdots \Theta_{i_{j}}^{i_{1}} \sum_{\ell=1}^{q-j}(-1)^{\ell-1} \frac{1}{\ell} \nabla_{i_{1}}\left(\Theta_{i_{j+1}}^{i_{j+2}} \cdots \Theta_{i_{j+\ell}}^{i_{j+1}}\right) P^{q-j}(\Theta) .
\end{gathered}
$$

In fact, we have

$$
\nabla_{i_{1}}\left(\sum_{i_{2}, \cdots, i_{j}=2}^{n} \Theta_{i_{2}}^{i_{3}} \cdots \Theta_{i_{j}}^{i_{2}}\right)=(j-1) \sum_{i_{2}, \cdots, i_{j}=2}^{n}\left(\nabla_{i_{1}} \Theta_{i_{2}}^{i_{3}}\right) \cdots \Theta_{i_{j}}^{i_{2}}
$$

and

$$
\begin{array}{r}
\nabla_{i_{1}}\left(\Theta_{i_{2}}^{i_{3}} \cdots \Theta_{i_{j}}^{i_{1}}\right)=\left(\nabla_{i_{1}} \Theta_{i_{2}}^{i_{3}}\right) \Theta_{i_{3}}^{i_{4}} \cdots \Theta_{i_{j}}^{i_{1}}+\sum_{k=3}^{j-1} \Theta_{i_{2}}^{i_{3}} \cdots \Theta_{i_{k-1}}^{i_{k}}\left(\nabla_{i_{1}} \Theta_{i_{k}}^{i_{k+1}}\right) \cdots \Theta_{i_{j}}^{i_{1}} \\
+\Theta_{i_{2}}^{i_{3}} \cdots \Theta_{i_{j-1}}^{i_{j}}\left(\nabla_{i_{1}} \Theta_{i_{j}}^{i_{1}}\right) .
\end{array}
$$


Using (4.11) in the first term on the right-hand side of (4.10) and using (4.12) in the second term of (4.10), we get

$$
\begin{gathered}
\sum_{i_{1}, i_{2}=2}^{n} X^{i_{1}} \nabla_{i_{1}} \Theta_{i_{2}}^{i_{2}} P^{q-2}(\Theta)+\sum_{j=3}^{q} \sum_{i_{1}, \cdots, i_{j}=2}^{n}(-1)^{j} X^{i_{1}}\left(\nabla_{i_{1}} \Theta_{i_{2}}^{i_{3}}\right) \cdots \Theta_{i_{j}}^{i_{2}} P^{q-j} \\
-\sum_{j=2}^{q} \sum_{i_{1}, \cdots, i_{j}=2}^{n}(-1)^{j} X^{i_{2}}\left(\nabla_{i_{1}} \Theta_{i_{2}}^{i_{3}}\right) \cdots \Theta_{i_{j}}^{i_{1}} P^{q-j}(\Theta) \\
-\sum_{j=2}^{q} \sum_{i_{1}, \cdots, i_{j}=2}^{n}(-1)^{j} \sum_{k=3}^{j-1} X^{i_{2}} \Theta_{i_{2}}^{i_{3}} \cdots \Theta_{i_{k-1}}^{i_{k}}\left(\nabla_{i_{1}} \Theta_{i_{k}}^{i_{k+1}}\right) \cdots \Theta_{i_{j}}^{i_{1}} P^{q-j}(\Theta) \\
-\sum_{j=2}^{q} \sum_{i_{1}, \cdots, i_{j}=2}^{n}(-1)^{j} X^{i_{2}} \Theta_{i_{2}}^{i_{3}} \cdots\left(\nabla_{i_{1}} \Theta_{i_{j}}^{i_{1}}\right) P^{q-j}(\Theta) \\
-\sum_{j=2}^{q} \sum_{i_{1}, \cdots, i_{j+\ell}=2}^{n}(-1)^{j} X^{i_{2}} \Theta_{i_{2}}^{i_{3}} \cdots \Theta_{i_{j}}^{i_{1}} \sum_{\ell=1}^{q-j}(-1)^{\ell+1}\left(\nabla_{i_{1}} \Theta_{i_{j+1}}^{i_{j+2}}\right) \cdots \Theta_{i_{j+\ell}}^{i_{j+1}} P^{q-j}(\Theta) .
\end{gathered}
$$

By using (4.4) on the third term of (4.13), the first three terms of (4.13) are cancelled out. Changing the indices in the last term of (4.13),

$$
\begin{aligned}
& -\sum_{j=2}^{q} \sum_{i_{1}, \cdots, i_{j+\ell}=2}^{n}(-1)^{j} X^{i_{2}} \Theta_{i_{2}}^{i_{3}} \cdots \Theta_{i_{j}}^{i_{1}} \sum_{\ell=1}^{q-j}(-1)^{\ell+1}\left(\nabla_{i_{1}} \Theta_{i_{j+1}}^{i_{j+2}}\right) \cdots \Theta_{i_{j+\ell}}^{i_{j+1}} P^{q-j}(\Theta) \\
& =-\sum_{j=2}^{q} \sum_{i_{1}, \cdots, i_{j}=2}^{n} \sum_{k=3}^{j-1}(-1)^{j-1} X^{i_{2}} \Theta_{i_{2}}^{i_{3}} \cdots \Theta_{i_{k-1}}^{i_{1}}\left(\nabla_{i_{1}} \Theta_{i_{k}}^{i_{k+1}}\right) \cdots \Theta_{i_{j}}^{i_{k}} P^{q-j}(\Theta) \\
& \quad-\sum_{j=2}^{q} \sum_{i_{1}, \cdots, i_{j}=2}^{n}(-1)^{j-1} X^{i_{2}} \Theta_{i_{2}}^{i_{3}} \cdots \Theta_{i_{j-1}}^{i_{1}}\left(\nabla_{i_{1}} \Theta_{i_{j}}^{i_{j}}\right) P^{q-j}(\Theta) .
\end{aligned}
$$

By using (4.4) in (4.14), we obtain

$$
\begin{aligned}
& -\sum_{j=2}^{q} \sum_{i_{1}, \cdots, i_{j+\ell}=2}^{n}(-1)^{j} X^{i_{2}} \Theta_{i_{2}}^{i_{3}} \cdots \Theta_{i_{j}}^{i_{1}} \sum_{\ell=1}^{q-j}(-1)^{\ell+1}\left(\nabla_{i_{1}} \Theta_{i_{j+1}}^{i_{j+2}}\right) \cdots \Theta_{i_{j+\ell}}^{i_{j+1}} P^{q-j}(\Theta) \\
& =\sum_{j=2}^{q} \sum_{i_{1}, \cdots, i_{j}=2}^{n} \sum_{k=3}^{j}(-1)^{j-1} X^{i_{2}} \Theta_{i_{2}}^{i_{3}} \cdots \Theta_{i_{k-1}}^{i_{1}}\left(\nabla_{i_{k}} \Theta_{i_{1}}^{i_{k+1}}\right) \cdots \Theta_{i_{j}}^{i_{k}} P^{q-j}(\Theta) \\
& \quad+\sum_{j=2}^{q} \sum_{i_{1}, \cdots, i_{j}=2}^{n}(-1)^{j-1} X^{i_{2}} \Theta_{i_{2}}^{i_{3}} \cdots \Theta_{i_{j}}^{i_{1}}\left(\nabla_{i_{j}} \Theta_{i_{1}}^{i_{j}}\right) P^{q-j}(\Theta) .
\end{aligned}
$$

By substituting (4.15) back into (4.13), the last three terms of (4.13) are cancelled out. Therefore, (4.13) is equal to 0 .

By Lemma 4.1, we have

$$
\int_{M} q \tilde{P}^{q}(\nabla X, \Theta, \cdots, \Theta) \wedge \omega_{M}^{n-q}=\int_{M} \operatorname{div}(Y) \wedge \omega_{M}^{n-q} .
$$


Theorem 4.1 (General divergence theorem). Given a holomorphic vector field with $(q-1, q-1)$-valued form $Y$ defined in Lemma 4.1, we have

$$
\int_{M} \operatorname{div}(Y) \wedge \omega_{M}^{n-q}=\int_{M} L_{Y}\left(\omega^{n-q}\right)=0 .
$$

Proof. Let $\tilde{Y}=\sum_{i_{1}=2}^{n} \sum_{j=2}^{q} \sum_{i_{2}, \cdots, i_{j}=2}^{n}(-1)^{j-1} X^{i_{2}} \Theta_{i_{2}}^{i_{3}} \cdots \Theta_{i_{j}}^{i_{1}} P^{q-j}(\Theta) \otimes \frac{\partial}{\partial z_{i_{1}}}$. We have $Y=\sum_{i_{1}=2}^{n} X^{i_{1}} P^{q-1}(\Theta) \otimes \frac{\partial}{\partial z_{i_{1}}}+\tilde{Y}$. Compute

$$
\begin{aligned}
\int_{M} L_{\tilde{Y}}\left(\omega^{n-q}\right)=(n-q) \int_{M} \sum_{i_{2}, \cdots, i_{j}=2}^{n} & (-1)^{j-1} X^{i_{2}} \Theta_{i_{2}}^{i_{3}} \cdots \Theta_{i_{j}}^{i_{1}} \\
& \wedge\left(\tilde{g}_{i_{1} \bar{\lambda}} d \bar{z}_{\lambda}\right) \wedge P^{q-j}(\Theta) \wedge \omega^{n-1-q} .
\end{aligned}
$$

On Kähler manifolds, we have

$$
\begin{aligned}
\sum_{i_{1}, \lambda=2}^{n} \Theta_{i_{j}}^{i_{1}} \wedge\left(\tilde{g}_{i_{1} \bar{\lambda}} d \bar{z}_{\lambda}\right) & =\frac{\sqrt{-1}}{2 \pi} \sum_{i_{1}, \alpha, \beta, \lambda=2}^{n} R_{i_{j} \bar{\lambda} \alpha \bar{\beta}} d z_{\alpha} \wedge d \bar{z}_{\beta} \wedge d \bar{z}_{\lambda} \\
& =\frac{\sqrt{-1}}{2 \pi} \sum_{i_{1}, \alpha, \beta, \lambda=2}^{n} R_{i_{j} \bar{\beta} \alpha \bar{\lambda}} d z_{\alpha} \wedge d \bar{z}_{\beta} \wedge d \bar{z}_{\lambda}=0 .
\end{aligned}
$$

Therefore, we obtain $\int_{M} L_{\tilde{Y}}\left(\omega^{n-q}\right)=0$. By the linearity of vector addition and (2.85), we have

$$
\begin{aligned}
\int_{M} L_{Y}\left(\omega^{n-q}\right) & =\int_{M} L_{P^{q-1}(\Theta) \otimes X}\left(\omega^{n-q}\right) \\
& =\int_{M} P^{q-1}(\Theta) \wedge L_{X}\left(\omega^{n-q}\right) \\
& =\int_{M} L_{X}\left(P^{q-1}(\Theta) \wedge \omega^{n-q}\right) .
\end{aligned}
$$

By (4.16), Lemma 4.1 and Lemma 2.6 we obtain

$$
\begin{aligned}
\int_{M} L_{Y}\left(\omega^{n-q}\right) & =\int_{M} L_{X}\left(P^{q-1}(\Theta) \wedge \omega^{n-q}\right) \\
& =q \int_{M} \tilde{P}^{q}(\nabla X, \Theta, \cdots, \Theta) \wedge \omega^{n-q} \\
& =\int_{M} \operatorname{div}(Y) \wedge \omega^{n-q} \\
& =0 .
\end{aligned}
$$

\section{ACKNOWLEDGEMENTS}

This paper is part of the author's Ph.D. thesis. The author thanks her advisor, Z. Lu, for his mathematical insights and assistance during the preparation of this paper. She also thanks Professor Paul for pointing out the effect of K-semistability on Bando-Futaki invariants for hypersurfaces. 


\section{REFERENCES}

[1] S. Bando. An obstruction for Chern class forms to be harmonic. Kodai Math. J., 29(3):337345, 2006. MR2278770 (2007j:32018)

[2] E. Calabi. Extremal Kähler metrics. II. In Differential geometry and complex analysis, pages 95-114. Springer, Berlin, 1985. MR780039 (86h:53067)

[3] X. X. Chen and G. Tian. Ricci flow on Kähler-Einstein surfaces. Invent. Math., 147(3):487544, 2002. MR 1893004(2003c:53095)

[4] W. Y. Ding and G. Tian. Kähler-Einstein metrics and the generalized Futaki invariant. Invent. Math., 110(2):315-335, 1992. MR1185586 (93m:53039)

[5] A. Futaki. An obstruction to the existence of Einstein Kähler metrics. Invent. Math., 73(3):437-443, 1983. MR718940 (84j:53072)

[6] A. Futaki. Kähler-Einstein metrics and integral invariants, volume 1314 of Lecture Notes in Mathematics. Springer-Verlag, Berlin, 1988. MR947341 (90a:53053)

[7] A. Futaki. Asymptotic Chow semi-stability and integral invariants. Internat. J. Math., 15(9):967-979, 2004. MR2106156 (2005g:32029)

[8] A. Futaki and S. Morita. Invariant polynomials of the automorphism group of a compact complex manifold. J. Differential Geom., 21(1):135-142, 1985. MR806707 (87h:32061)

[9] P. Griffiths and J. Harris. Principles of algebraic geometry. Wiley-Interscience [John Wiley \& Sons], New York, 1978. Pure and Applied Mathematics. MR507725 (80b:14001)

[10] S. Kobayashi. On compact Kähler manifolds with positive definite Ricci tensor. Ann. of Math. (2), 74:570-574, 1961. MR0133086 (24:A2922)

[11] N. C. Leung. Bando Futaki invariants and Kähler Einstein metric. Comm. Anal. Geom., 6(4):799-808, 1998. MR 1664891 (2000d:32043)

[12] Z. Lu. On the Futaki invariants of complete intersections. Duke Math. J., 100(2):359-372, 1999. MR:1722959 (2000h:32033)

[13] Z. Lu. $K$ energy and $K$ stability on hypersurfaces. Comm. Anal. Geom., 12(3):601-630, 2004. MR2128605 (2006m:32024)

[14] D. H. Phong and J. Sturm. The Futaki invariant and the Mabuchi energy of a complete intersection. Comm. Anal. Geom., 12(1-2):321-343, 2004. MR2074881(2005c:32030)

[15] G. Tian. The K-energy on hypersurfaces and stability. Comm. Anal. Geom., 2(2):239-265, 1994. MR 1312688 (95m:32030)

[16] G. Tian and X. Zhu. A new holomorphic invariant and uniqueness of Kähler-Ricci solitons. Comment. Math. Helv., 77(2):297-325, 2002. MR.1915043 (2003i:32042)

[17] M. Yotov. Generalized Futaki invariant of almost Fano toric varieties, examples. Preprint, 1999.

Tims, Department of Mathematics, National Taiwan University, Taipei, Taiwan 106

E-mail address: cjliu4@ntu.edu.tw 